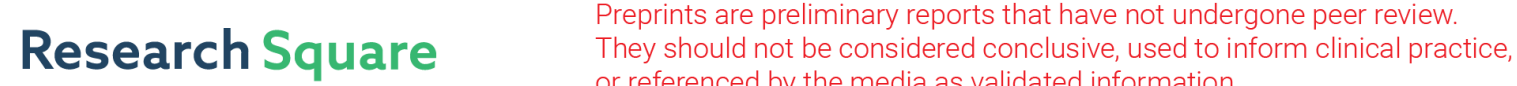 or referenced by the media as validated information. \\ Tacholess Rotational Speed Estimation via The Integration of Continuous Wavelet Transform and Time-Dependent Autoregression
}

\section{Ran Zhang}

China Agricultural University

Xingxing Liu ( $\square$ liuxingxing56285@163.com )

China Agricultural University

\section{Yongjun Zheng}

China Agricultural University

Haotun LV

China Agricultural University

\section{Baosheng Li}

China Agricultural University

\section{Shenghui Yang}

China Agricultural University

Yu Tan

China Agricultural University

\section{Research}

Keywords: Rotating Speed Estimation, Autoregressive Modelling, Wavelet Transform, Wavelet Covariance

Posted Date: September 16th, 2020

DOl: https://doi.org/10.21203/rs.3.rs-75408/v1

License: (c) (i) This work is licensed under a Creative Commons Attribution 4.0 International License.

Read Full License 


\title{
Tacholess Rotational Speed Estimation via the Integration of Continuous Wavelet Transform and Time-dependent Autoregression
}

\author{
Ran Zhang, Xingxing Liu*, Yongjun Zheng, Haotun Lv, Baosheng Li, Shenghui Yang, Yu Tan \\ College of Engineering, China Agricultural University, Beijing 100083, China \\ liuxingxing56285@163.com
}

\begin{abstract}
Speed estimation is crucial to monitor the conditions of rotational machinery. Most speed measurements are carried out by installing encoders or tachometers inside the machines. In many cases, such method could be cumbersome or even inaccessible. This paper proposes a vibration-based speed estimation method. The vibration sensors are often cheaper and easier to install than angle encoders. In the proposed method, the continuous wavelet transform (CWT) is used as a preprocessing technique to extract the signal of importance. Then, the time-varying autoregressive (TAR) model is applied to analyze the rotational frequency. Additionally, the paper presents a fast algorithm for implementation. The proposed method is validated by both synthetic and empirical data.
\end{abstract}

Keywords: Rotating Speed Estimation; Autoregressive Modelling; Wavelet Transform; Wavelet Covariance;

\section{Introduction}

The rotational speed estimation of machinery is essential to condition monitoring because of its significant impacts on emission, fuel consumption, noise, dynamics and malfunction diagnosis [1] [2] [3] [4] [5]. In many cases, the direct measurement of rotational speed could be extremely difficult and costly [6]. This is because the encoders or tachometer needs to be positioned on or close to the rotational components and the working environment is harsh [7]. Therefore, the estimation of rotating speed from vibration signal has been received tremendous attentions.

The frequency contents of vibration signals of rotation machines are very complex [1] [8] [9] [10]. Due to the low signal to noise ratio (SNR) and various undesired frequency components, the accurate estimation of rotational speed from vibration is still a great challenge to existing applications. Further, many rotating machines operate at fluctuated speed which produces non-stationary signals [11]. Hence, the speed estimating requires robust and adaptive techniques to deal with the aforementioned challenges.

Numerous achievements have been accomplished in this area of vibration analysis. K.C. Gryllias and I.A. Antoniadis [12] present a complex shifted Morlet wavelets based instantaneous speed estimation algorithm. Its advantage is the free selection of the wavelet's center frequency and bandwidth, making the algorithm adaptive to signal. Nonetheless, a limited number of center frequency selected could be too coarse to identify the speed variation. An automatic speed estimation for gear pair is proposed in [13], combining the singular spectrum decomposition and phase coupling analysis. This method depends solely on the harmonics of the gear-mesh frequency and lacks of noise suppression. H. Lin and K. Ding [14] provides a four stroke engine speed measurement. Its principle is that the half harmonics of the torque of combustion engine can be explicitly recognized via the spectrum autocorrelation. One problem with the method is the highest peak results from the autocorrelation may not always be the half harmonic order.

Parallel, the joint use of filter method and parametric modelling is an alternative way for speed estimation. Its main advantages are the adaptability of filterbank setting and high estimating resolution comes from the modelling [15]. Hence, such concept has great potentials and should be open studied. However, quite few literatures with respect to the concept are found in measuring the rotational speed. One research can be cited is [16]. The paper proposes an instantaneous frequencies estimation based on the combination of complex Morlet wavelet and ESPRIT. By setting appropriate wavelet filterbank, the ESPRIT is able to recover the rotating frequency without interference of undesired components and noise. The restrictions of using this technique are the non-strictly analytic properties of the complex Morlet wavelet and the intensive computation of subspace algorithm [17] [18].

In this paper, we propose a fusion algorithm combing the CWT and TAR to estimate the rotational speed. The CWT works as bandpass filters to suppress the noise and undesired frequency component while retaining the signal of importance. Thus, the TAR built by the wavelet coefficients is adaptable. Additionally, a fast implementation of the algorithm is presented to reduce the computational cost. Compared with [16], the main novelties are the applicability of a wide range of wavelet families and the efficiency in computation. The estimating performance is validated via both synthetic and empirical data. 


\section{Methods}

The principle of the proposed algorithm is using the wavelet coefficients to build the Modified Yule-Walker (MYW) equation. The wavelet transform is essentially a bandpass filter of uniform shape and varying location and width [19]. By appropriating setting the CWT filters, the model becomes adaptive to the signal. Once the MYW equation is created, we solve it by total least square (TLS) to obtain the model's parameters. Finally, the rotating frequency is estimated via Cadzow's method.

It is worth noting that the TAR is the moving window version of classical autoregressive (AR) model and is detailed in [15]. For simplicity, we assume the described data to be windowed. Thus, the TAR is reduced to AR in this section.

The proposed algorithm is mainly divided into two parts:

I. Autocovariance sequence (ACS) of CWT coefficients (fast implementation);

II. Solve the model via the ACS based MYW equation and estimate the frequency by Cadzow method.

\subsection{ACS of CWT coefficients}

The proposed algorithm uses the ACS to build the MYW equation. In this subsection, the CWT is briefly described first; then, the ACS of wavelet coefficients is introduced.

The CWT of signal $f$ at scale $s$ and time $t$ can be expressed as:

$$
w(t, s)=\frac{1}{s} \int_{-\infty}^{+\infty} f(\tau) \psi^{*}\left(\frac{\tau-t}{s}\right) d \tau
$$

where * represents the complex conjugate and $\psi$ is the mother wavelet. $1 / s$ is the amplitude normalization factor. By modifying the scale parameter, the wavelet is able to construct desired bandpass filters [20].

Summing over a subset of the scales in (1), the filtered signal is then obtained:

$$
W(t)=\sum_{i=i 1}^{i 2} w\left(t, s_{i}\right)
$$

Such filter has a frequency response given by the sum of the wavelet functions between scales i1 and i2, discriminating frequency components outside its bandwidth.

Next, the ACS of the wavelet coefficients is calculated by:

$$
r_{\psi}(k)=E\left\{W(t) W^{*}(t-k)\right\}
$$

where $E\{$.$\} denotes the expectation operator and the sequence is assumed to be zero mean. Once the ACS is obtained, the$ AR model can be solved from the MYW equation:

$$
r_{\psi}(k)+\sum_{p=1}^{P} a_{p} r_{\psi}(k-p)=0
$$

where $\alpha$ is the AR parameter and p represents the AR order. The model is adaptive to the analyzed signal because the $r_{\psi}(k)$ is calculated from the filtered signal.

\subsection{Fast Implementation}

The AR model built directly from (3) is computationally expensive. Compared with the convolution operation in time domain, it is considerable faster to do the calculation in frequency domain. The paper provides a Fourier transform (FT) based algorithm to calculate the $r_{\psi}(k)$ in order to efficiently create the MYW equation. Its principle is that the $r_{\psi}(k)$ is essentially a wavelet transform of the ACS of the raw signal [21]. This can be calculated in Fourier space; the wavelet used is a new wavelet resulting from the autocovariance of the original wavelet. The fast algorithm is illustrated in Fig. 1.

Let $\odot$ denotes the covariance operator and $\otimes$ denotes the convolution operator respectively. The fast implementation procedures are as follows: 
Calculate the new wavelet in frequency domain. The new wavelet, termed $\Psi$, is obtained through the autocovariance of the original wavelet. A simple way of the calculation derives from the fact that many wavelets have symmetric or antisymmetric structure. By combining the correlation property $f(t) \odot f(t)=f(-t) \otimes f(t)$, the new wavelet is then:

$$
\begin{gathered}
\Psi(t)=\psi(t) \otimes \psi(t) \quad \psi(t) \text { is symmetric } \\
\Psi(t)=-\psi(t) \otimes \psi(t) \quad \psi(t) \text { is anti-symmetric }
\end{gathered}
$$

Equation (5) and (6) leads to:

$$
\hat{\Psi}(\omega)=\hat{\psi}(\omega) \times \hat{\psi}(\omega)
$$

where $\hat{\Psi}$ and $\hat{\psi}$ are the Fourier transform of $\Psi$ and $\psi$ respectively. Thus, the new wavelet is obtained.

Taking the $\mathrm{m}^{\text {th }}$ order derivatives of Gaussian wavelets (DOG) as an example. Its expression in frequency domain is [22]:

$$
\hat{\psi}(\omega)=\left(-i^{m}\right)(\omega)^{m} e^{-\frac{(\omega)^{2}}{2}}
$$

The new wavelets of the autocovariance of the DOG is then given by:

$$
\hat{\Psi}(\omega)=\hat{\psi}(\omega) \times \hat{\psi}(\omega)=\left(i^{2 m}\right)(\omega)^{2 m} e^{-(\omega)^{2}}
$$

Calculate the autocovariance of raw signal in frequency domain. The autocovariance can also be calculated via FT. For the zero-mean sequence, the autocovariance and correlogram are a Fourier transform pair:

$$
\mathcal{F}\{r(k)\}=|\hat{f}(\omega)|^{2}
$$

where $r(k)$ is the autocovariance of the sequence; $\hat{f}$ is the Fourier transform of the sequence; and $\mathcal{F}$ represents the Fourier transform operator.

Calculate the ACS of wavelet coefficients. By multiplying equation (10) with the new wavelet $\hat{\Psi}(\omega)$, the wavelet transform of $r(k)$ is done in frequency domain. And, the subset of scales from $i_{1}$ to $i_{2}$ should be summed. Finally, $r_{\psi}(k)$ can be calculated by:

$$
r_{\psi}(k)=\mathcal{F}^{-1}\left\{\sum_{i 1}^{i 2}\left[|\hat{f}(\omega)|^{2} \times \hat{\Psi}_{i}\right]\right\}
$$




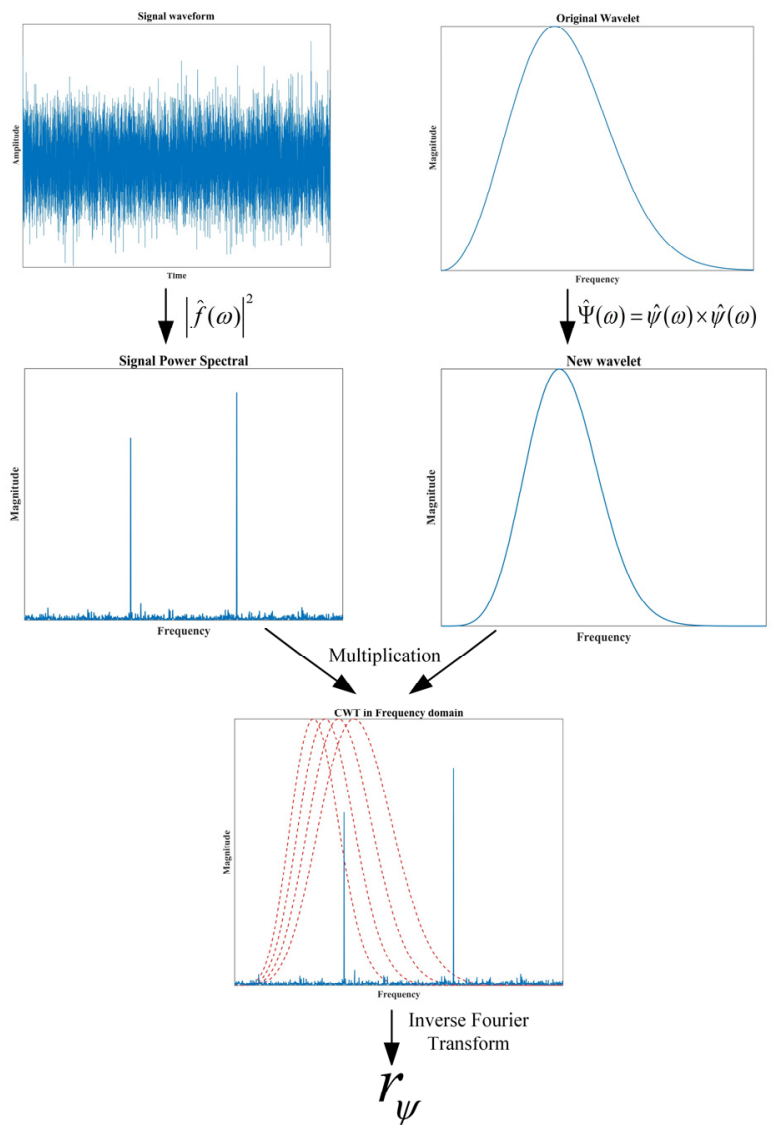

Fig. 1 Illustration of the fast implementation

\subsection{Frequency Estimation from the MYW equation}

Many effective algorithms have been developed to solve the AR model [18] [23]. One of the most commonly used techniques is the MYW method; it is often complemented with the TLS. After solving the model, the rotation frequency is then derived from the Cadzow's method. The frequency estimation consists of the following steps:

Step 1: Create the MYW equation. By setting the appropriate AR order $P$ and MA order $Q$, the MYW equation can be created:

$$
\sum_{p=1}^{P} a_{p} r_{\psi}(l-p)=-r_{\psi}(l) \quad l=Q+1, \ldots, Q+P
$$

Step 2: AR parameters estimation via TLS. The AR parameters $a_{p}$ are then approximated by TLS. This is done by performing the singular value decomposition (SVD) on (12), resulting in a diagonal matrix $(\Sigma)$ of non-zero diagonal elements (singular values):

$$
r_{\psi}(l)+\sum_{p=1}^{P} a_{p} r_{\psi}(l-p)=U \cdot \Sigma \cdot V^{T}
$$

where $\mathrm{U}: l \times l, \Sigma: l \times(P+1), V:(P+1) \times(P+1)$.

Next, partition the $V$ corresponding to the $\hat{p}$ largest singular value:

$$
\left[\begin{array}{ll}
V_{11} & V_{12} \\
V_{21} & V_{22}
\end{array}\right]
$$

where the dimension is $V_{12}:(P+1-\hat{p}) \times(P+1-\hat{p})$ and $V_{22}: \hat{p} \times(P+1-\hat{p})$. The AR parameters are solved by: 


$$
a_{p}=-V_{22} V_{22}^{-1}
$$

Step 3: Spectral estimation by Cadzow's method. The Cadzow assume the power spectral density (PSD) of AR model can be expressed as [24]:

$$
P\left(e^{j \omega}\right)=\sum_{p=1}^{\infty} \rho(k) e^{-j \omega k}+\sum_{p=1}^{\infty} \rho(-k) e^{j \omega k}
$$

where $\rho(k)=\rho(-k)$ and can be obtained from the covariance of the signal:

$$
\rho(k)= \begin{cases}\frac{1}{2} r(k), & k=0 \\ r(k), & \text { others }\end{cases}
$$

Once the PSD is obtained, the rotating frequency is derived from the expectation of the PSD at every time interval.

\section{Results and Discussion}

In this section, the proposed algorithm is applied to both synthetic and empirical signals. Further, two other frequency estimation methods are presented for comparison purpose. One is the CWT which estimate the frequency by taking the expectation of the scalogram. In the analysis, the CWT shares the same wavelet parameters with the proposed algorithm. Another method is first conditional spectral moment method, termed FCSM. It is a common application that estimates the instantaneous frequency as the first conditional spectral moment of the time-frequency distribution of the signal [25].

\subsection{Synthetic Signal}

The synthetic signal consists a non-linear chirp with "instantaneous" frequency that varies sinusoidally between $120 \mathrm{~Hz}$ to $280 \mathrm{~Hz}$. Also, a white Gaussian noise is embedded into the signal with a significant SNR 10dB. The sampling frequency is set to be $5000 \mathrm{~Hz}$. The signal is depicted in Fig. 2.

The wavelet is the generalized Morse wavelets with $\gamma=3$ and $\beta=20$ which is defined as [26]:

$$
\psi(\omega) \triangleq 2\left(\frac{e \gamma}{\beta}\right)^{\beta / \gamma} \omega^{\beta} e^{-\omega^{\gamma}} \times\left\{\begin{array}{cc}
1 & \omega>0 \\
\frac{1}{2} & \omega=0 \\
0 & \omega<0
\end{array}\right.
$$

The wavelet filterbank is shown in Fig. 3 that has $-3 \mathrm{~dB}$ bandwidth between $100 \mathrm{~Hz}$ to $300 \mathrm{~Hz}$. The rectangular window has 40 samples and steps forward every 20 samples.
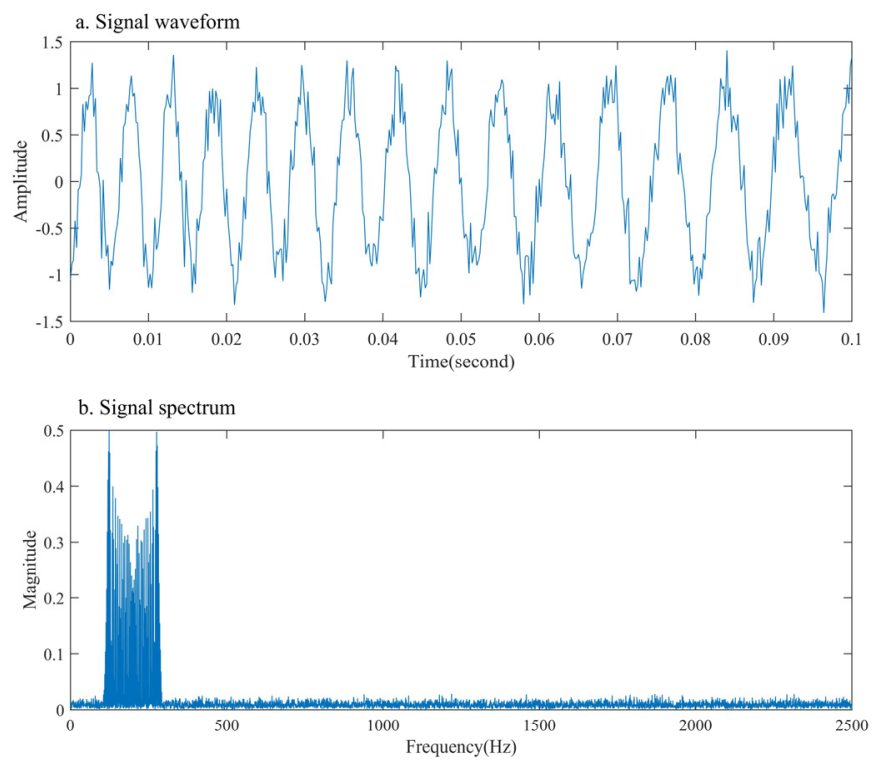

Fig. 2 The synthetic signal. a. signal waveform. b. signal spectrum. 


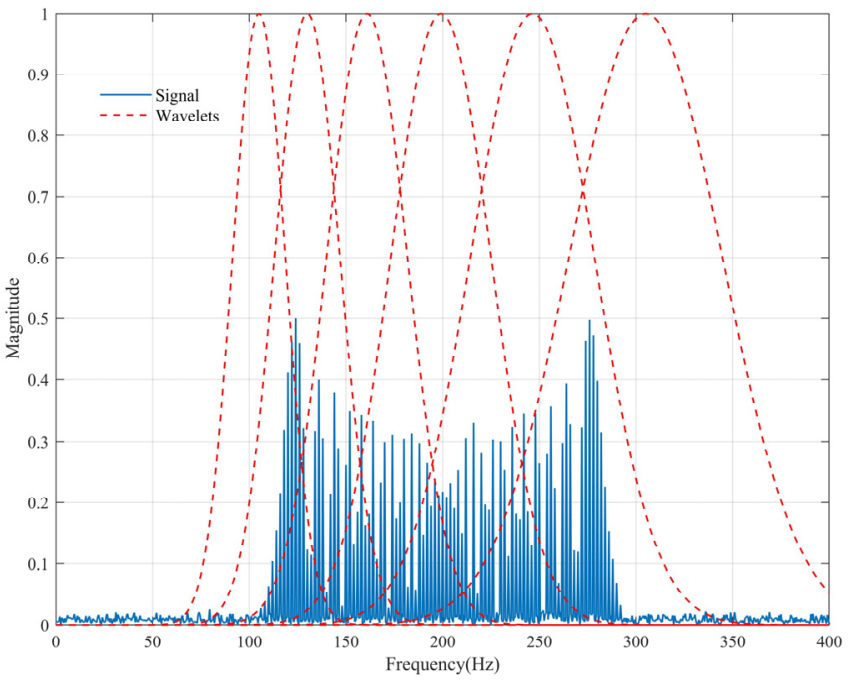

Fig. 3 The filterbank.

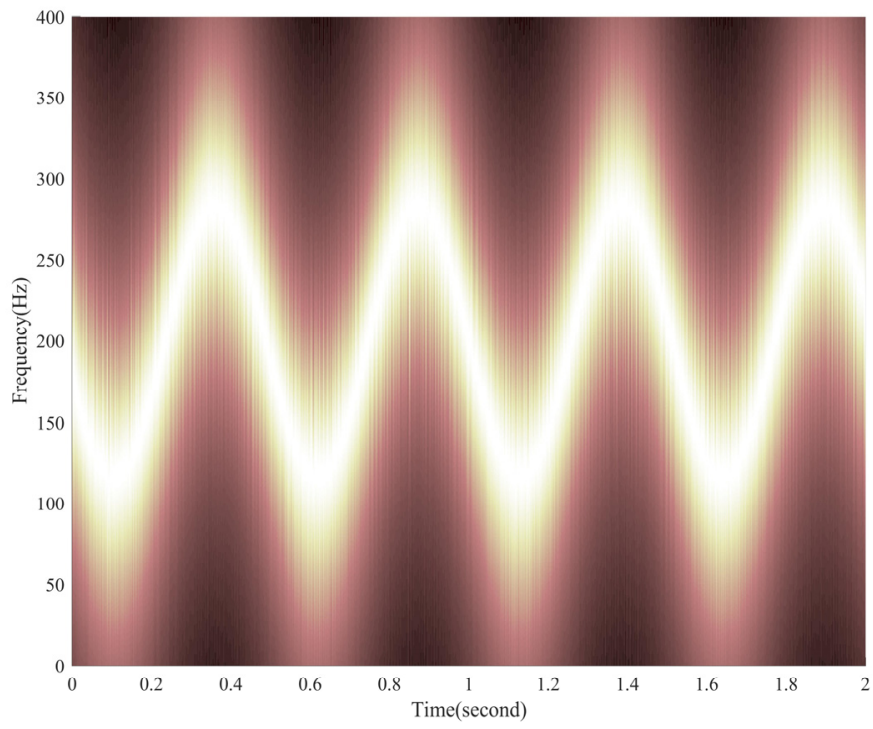

Fig. 4 PSD of the signal. 


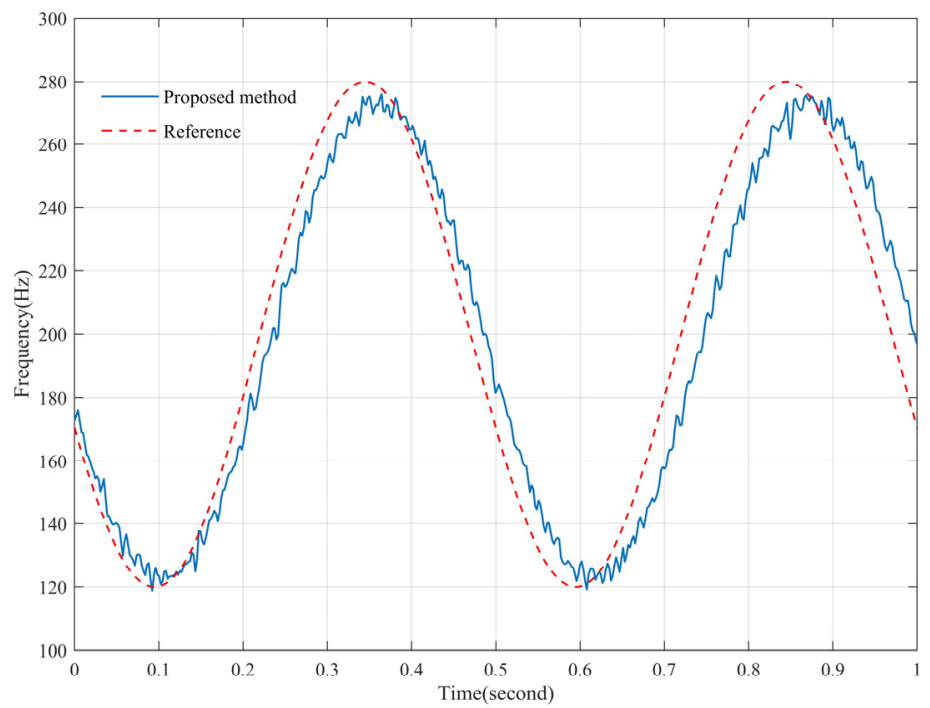

Fig. 5 Estimated result of the proposed method.

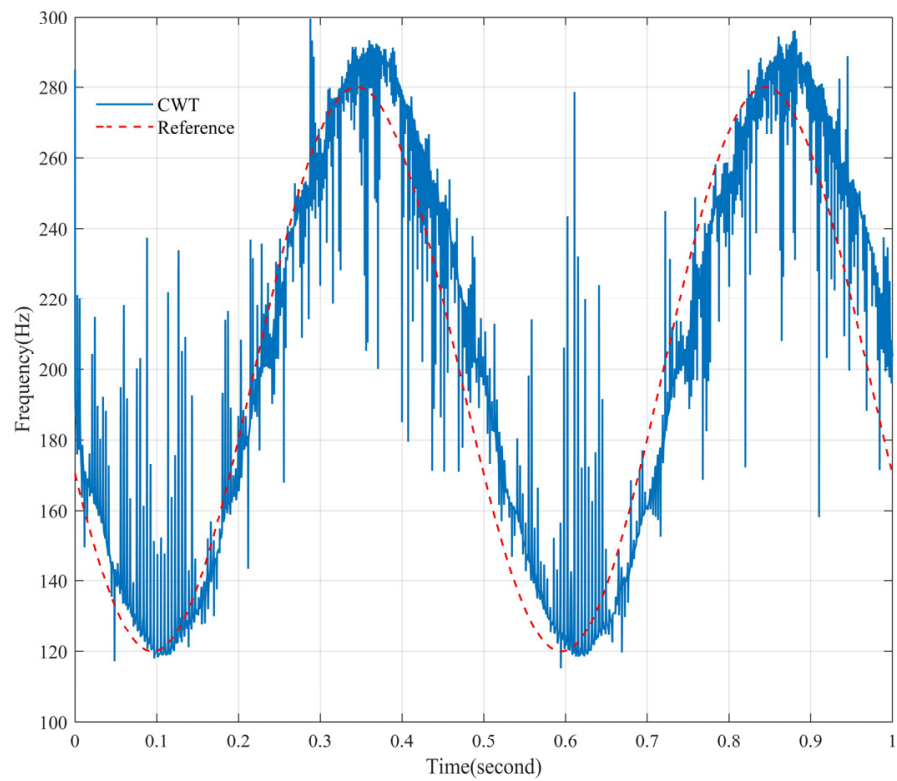

Fig. 6 Estimated result of the CWT. 


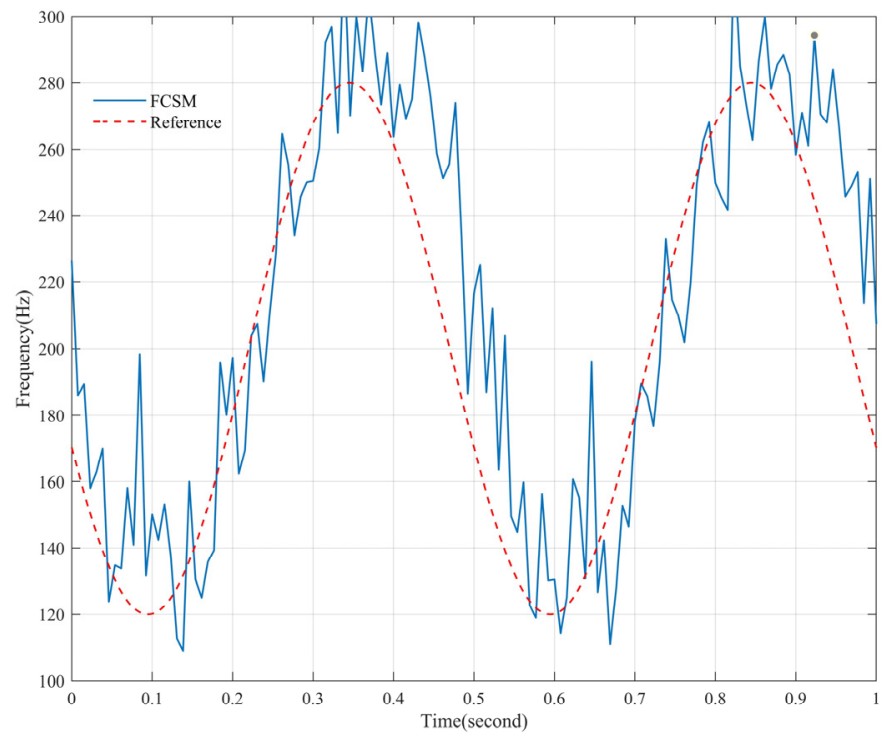

Fig. 7 Estimated result of the FCSM.

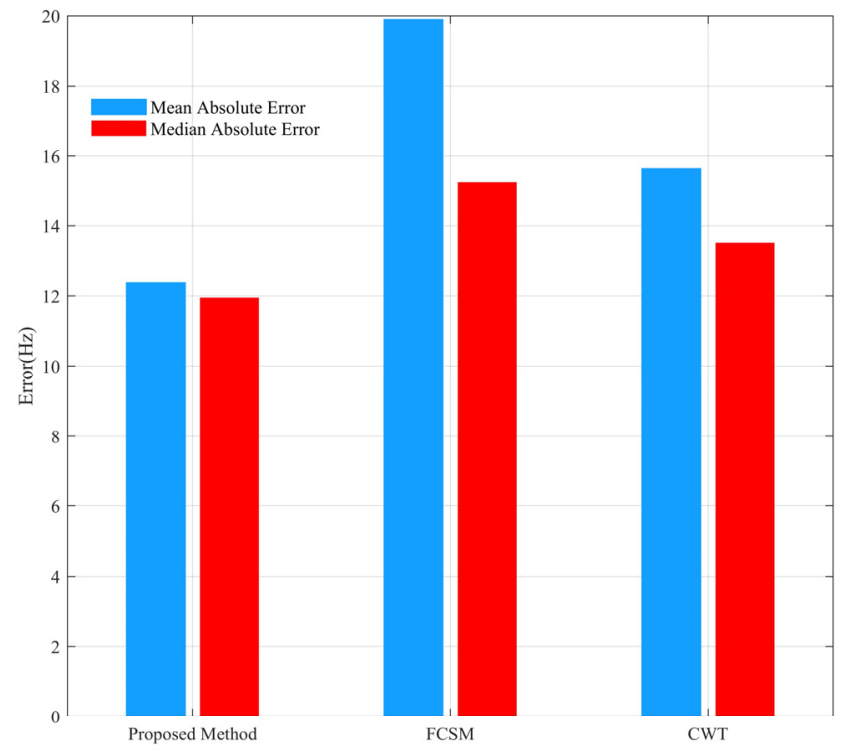

Fig. 8 Mean and median absolute errors.

The PSD and estimated results are presented in Fig. 4 and Fig. 5 respectively. Also, the estimation of CWT and FCSM are depicted in Fig. 6 and Fig. 7. The reference frequency is marked in red dashed line. Obviously, the proposed method demonstrates high estimating accuracy compared with the CWT and FCSM. The use of six wavelets in CWT lacks of enough 'scale resolution', leading to the drastically large variance. The FCSM presents large fluctuations in comparison to the proposed method because of its non-adaptability, being corrupted by the noise. The mean and median absolute errors are shown in Fig. 8.

The wavelet filter makes the proposed algorithm adaptive to the signal, suppressing undesired frequency components out side its bandwidth. Further, the AR model offers a high frequency resolution that enables the high accuracy in estimation.

\subsection{Aircraft Engine Data}

In this case, the data is the aircraft engine vibration provided by [27]. The signal represents a slow acceleration progress from idle to full power as displayed in Fig. 9. An accelerometer and a tachometer are installed to record the vibration 
signal and the angular speed respectively. The vibration signal is initially sampled at $50 \mathrm{~K} \mathrm{~Hz}$ and then down sampled to $31.2 \mathrm{~K} \mathrm{~Hz}$.

The generalized Morse wavelet ( $\gamma=3$ and $\beta=20$ ) is used to set the filters. Owing to the engine characteristics, the wavelets are set to cover the frequency band $175 \mathrm{~Hz}$ to $260 \mathrm{~Hz}$. Hence, the undesired components and noise outside the band are suppressed. The wavelet filters are presented in Fig. 10. The window length is 4000 samples with 50\% overlap.
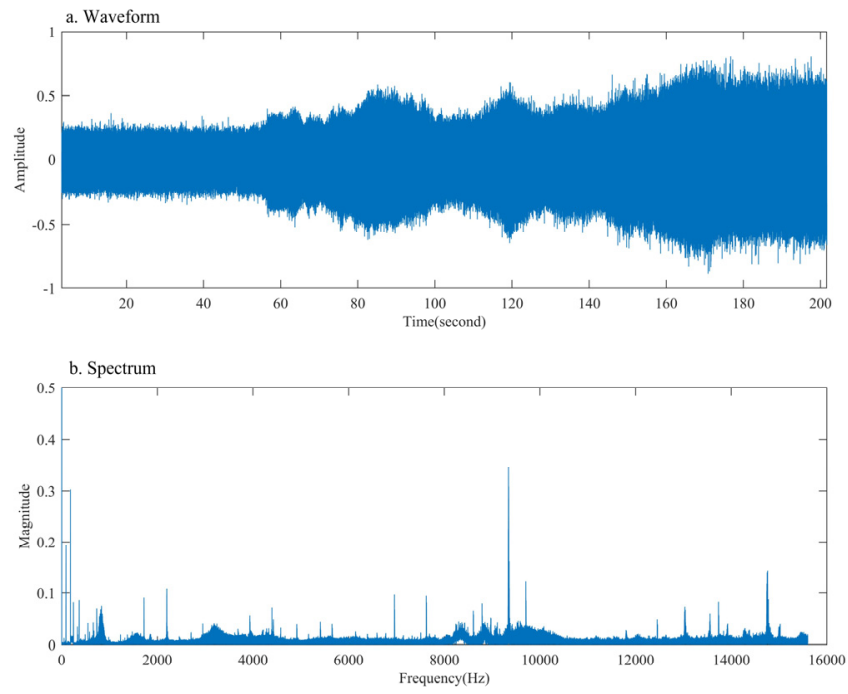

Fig. 9 The vibration signal. a. signal waveform. b. signal spectrum.

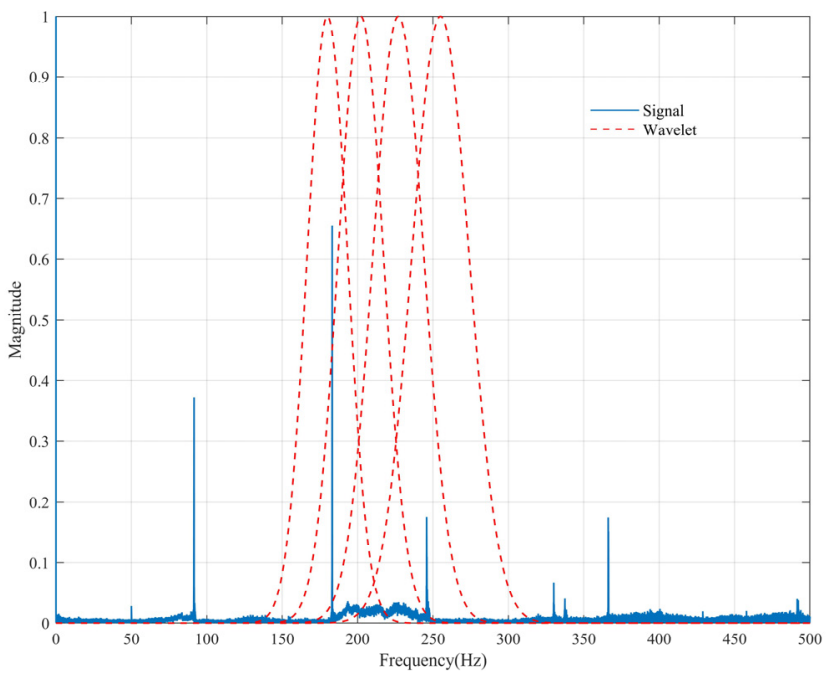

Fig. 10 wavelet filterbank. 


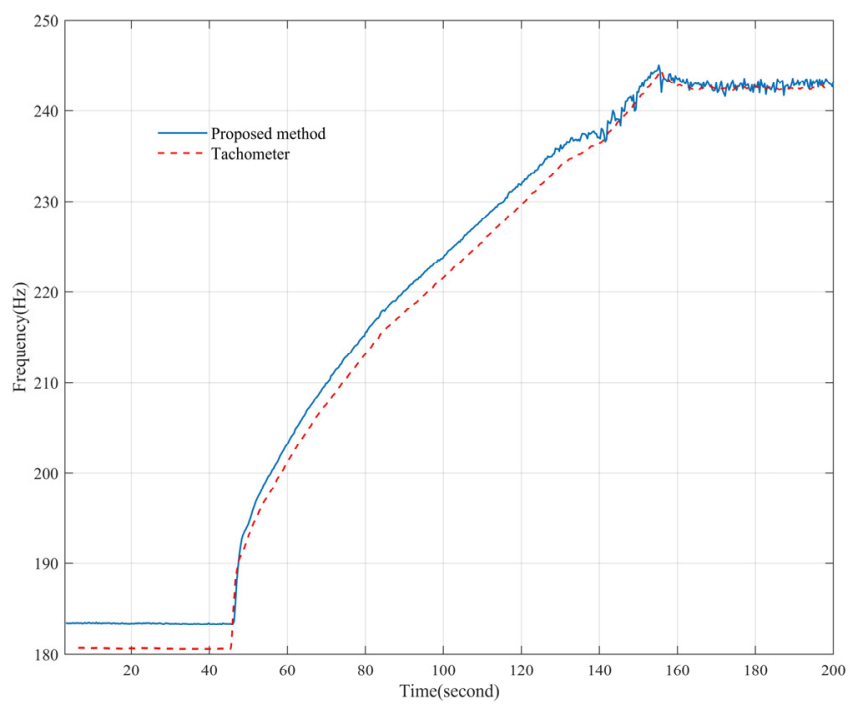

Fig. 11 Estimated speed frequency of proposed method.

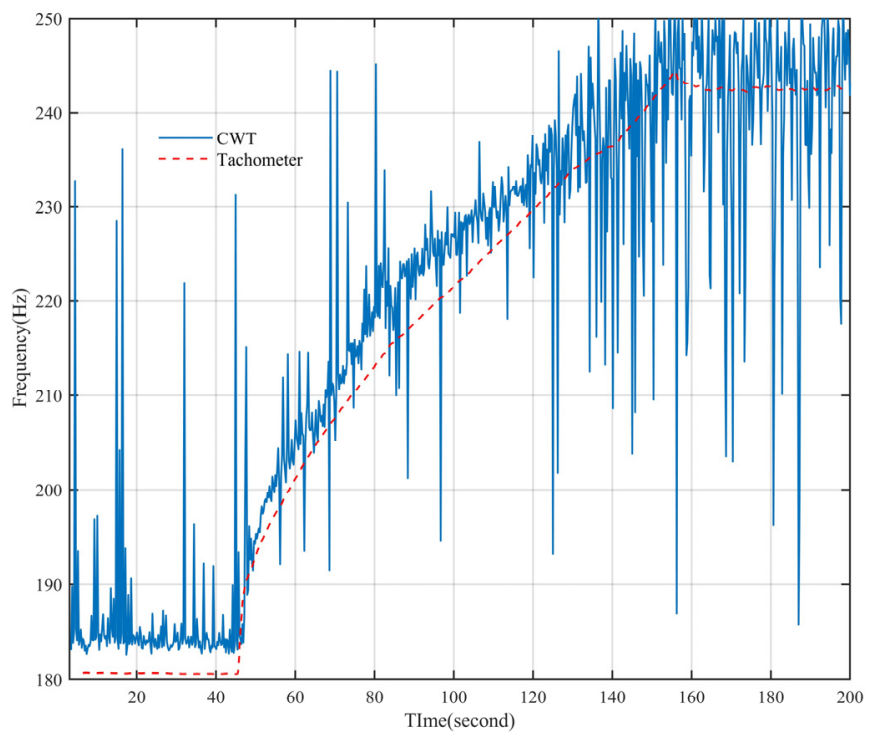

Fig. 12 Estimated speed frequency of CWT. 


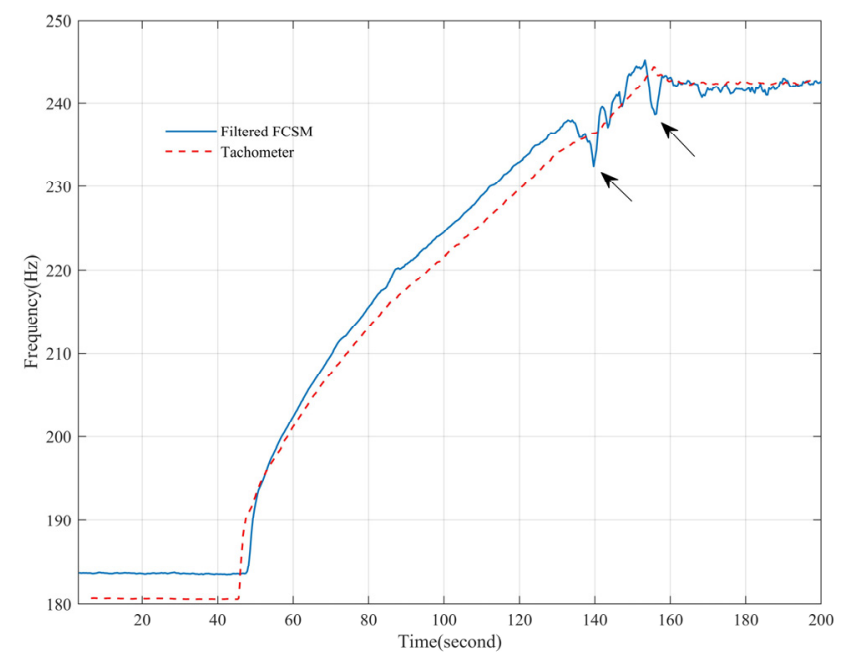

Fig. 13 Estimated speed frequency of Filtered FCSM.

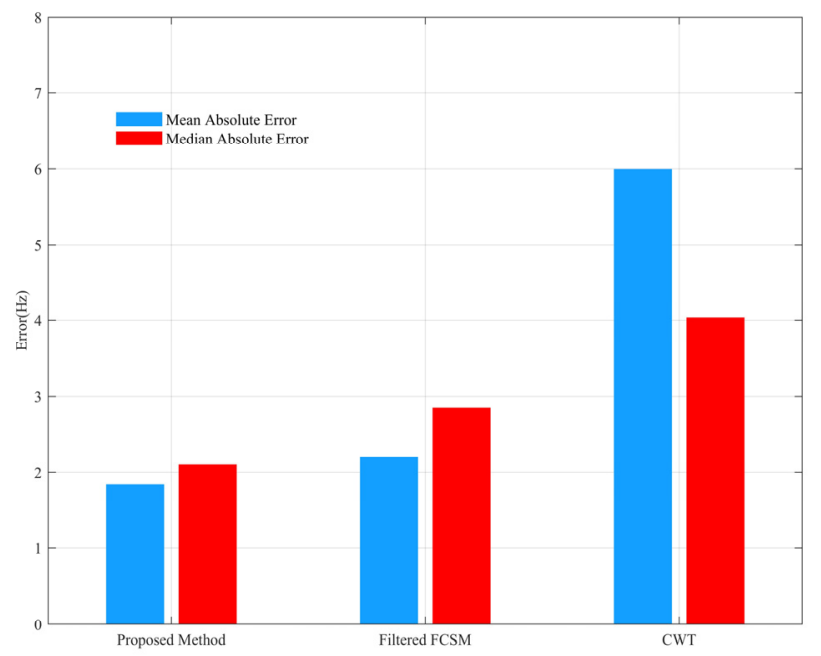

Fig. 14 Mean and median absolute errors.

The estimated result is depicted in Fig. 11. During the idle stage, the estimated frequency remains at $183.5 \mathrm{~Hz}$ with a mean error of $2.8 \mathrm{~Hz}$. This is actually an expected result since the tachometer is not mounted on the main shaft where the accelerometer is closed to. It is also seen that the sudden increase in rotational speed around $45 \mathrm{~s}$ strains the estimated accuracy due to the smearing of harmonics. Due to the increased SNR when the engine reaches to its full power, the estimated frequency becomes a bit choppy but neglectable. Overall, the proposed method effectively recovers the rotating speed from vibration signal with high accuracy.

Fig. 12 illustrates the CWT estimation in which the engine speed can hardly be recognized. This is mainly due to the four wavelet scales are too coarse to identify the frequency content. The FCSM is presented in Fig. 13. In this case, a bandpass filter is applied first with passband range $[175 \mathrm{~Hz} 260 \mathrm{~Hz}]$. This is because the raw estimation of FCSM is significantly corrupted by the strong background noise, making the comparison meaningless. Its estimating accuracy is inferior to the proposed method. Two main distortions are pointed out in Fig. 13. The mean and median absolute error of the three methods are presented in Fig. 14.

\section{Conclusion}

The paper presents a vibration signal based rotational speed estimation method that integrates CWT and AR model. By combining the advantages of adaptive filter and parametric modeling, the speed frequency can be recovered with good accuracy. It should be noting that the direct measurement approaches (e.g. tachometer) is still superior in accuracy. 
Nonetheless, the installations of such devices required to be on the shaft of interest which can be unfeasible. On the other hand, the vibration sensors are often cheaper and easier to instrument than tachometers. Hence, the proposed method is an alternative way to effectively measure the rotational speed.

\section{List of abbreviations}

\begin{tabular}{|cc|cc|}
\hline CWT & Continuous Wavelet Transform & TAR & Time-dependent Autoregression \\
AR & Autoregression & MA & Moving Average \\
SNR & Signal to Noise Ratio & MYW & Modified Yule-Walker Method \\
TLS & Total Least Square & ACS & Autocovariance Sequence \\
FT & Fourier Transform & DOG & Derivatives of Gaussian Wavelet \\
PSD & Power Spectral Density & FCSM & First Conditional Spectral Moment Method \\
\hline
\end{tabular}

\section{Declaration}

Ethics approval and consent to participate:N/A

Consent for publication:N/A

Availability of data and material: Aircraft engine vibration data is available online (DOI: 10.17632/s8jrrnnj9g.1)

Competing interests: The authors declare that they have no known competing financial interests or personal relationships that could have appeared to influence the work reported in this paper.

Funding: This research is supported by the National Key R\&D Program of China (Grant No. 2016YFD0700302).

Authors' contributions: Ran Zhang (method, validation, code); Xingxing Liu(method, validation,code), Yongjun Zheng(method); Haotun Lv(method); Baosheng Li(validation, code); Shenghui Yang(validation,code); Yu Tan(method, validation)

Acknowledgement:N/A

\section{References}

[1] S. Delvecchio, P. Bonfiglio and F. Pompoli, "Vibro-acoustic condition monitoring of Internal Combustion Engines: A critical Review of existing techniques," Mechanical Systems and Signal Processing, vol. 99, pp. 661-683, 2017.

[2] K. O. Farag, Y. S. Mohamed and A. E. Samir, "Time and frequency analyses of dual-fuel engine block vibration," Fuel, vol. 203, pp. 884-893, 2017.

[3] M. Gerhard, H. Martin, W. Helmut, B. Josef and G. Andreas, "Energy consumption in cultivating and ploughing with traction improvement system and consideration of the rear furrow wheel-load in ploughing," Soil \& Tillage Researchj, vol. 134, pp. 56-60, 2013.

[4] D. Taraza, "Statistical Correlation Between the Crankshaft's Speedf Variation and Engine Performance-Part I:Theoretical Model," ASME Journal of Engineering for Gas Turbines and Power, vol. 125, pp. 791-796, 2003.

[5] J. Antoni, J. Daniere and F. Guillet, "Effective vibration analysis of IC engines using cyclostationarity. Part I-A metholology for condition monitoring," Journal of Sound and VIbration, vol. 257, no. 5, pp. 815-837, 2001.

[6] P. Cedric, L. Quentin, A. Jerome, L. Peter, d. John, L. Steven and H. Jan, "Review and comparison of tacholess instantaneous speed estimation methods on experimental vibration data," Mechanical Systems and Signal Processing, vol. 129, pp. 407-436, 2019.

[7] Y. Li, F. Gu, G. Harris, A. Ball, N. Bennett and K. Travis, "The measurement of instantaneous angular speed," Mechanical Systems and Signal Processing, vol. 19, pp. 897-920, 2005. 
[8] Z. Xiuliang, C. Yong, W. Limei and J. Shaobo, "Real time identification of the internal combustion engine combustion parameters based on the vibration velocity signal," Journal of Sound and VIbration, vol. 390, pp. $205-$ $217,2016$.

[9] T.-. A. Ahmad and M. Alireza, "Fault detection of injectors in disel engines using vibration time-frequency analysis," Applied Acoustics, vol. 143, pp. 48-58, 2018.

[10] C. Paul, Y. Sireesha, C. C.L.Philip and A. Arturo, "Optimization of Sensor Locations and Sensitivity Analysis for Engine Health Monitoring Using Minimum Interference Algorithm," EURASIP Journal on Advances in Signal Processing, vol. 2008, p. 280346, 2007.

[11] G. Hongrui, H. Dong, X. Songtao and C. Xuefeng, "Vibration signal correction of unbalanced rotor due to angular speed fluctuation," Mechanical Systems and Signal Processing, vol. 107, pp. 202-220, 2018.

[12] C. G. Konstantinos and A. A. Ioannis, "Estimation of the instantaneous rotation speed using complex shifted Morlet wavelets," Mechanical Systems and SIgnal Processing, vol. 38, pp. 78-95, 2012.

[13] L. R. B. Mario, E. H. M. Fidel, C. G. M. Julio and P. M. Evelio, "Tacho-less automatic rotational speed estimation (TARSE) for a mechanical system with gear pair under non-stationary conditions," Measurement, vol. 145, pp. 480-494, 2019.

[14] L. Hinbin and D. Kang, "A new method for measuring engine rotational speed based on the vibration and discrete spectrum correction," Measurement, vol. 46, pp. 2056-2064, 2013.

[15] M. Spiridonakos and S. Fassois, "Non-stationary random vibration modelling and analysis via functional series time-dependent ARMA (FS-TARMA) models: A critical survey," Mechanical Systems and Signal Processing, vol. 47, pp. 175-224, 2013.

[16] A. A. Ioannis, T. Y. Christos, C. G. Konstantions and I. R. Konstantinos, "IFESIS: Instantaneous frequencies estimation vis subspace invariance properties of wavelet structures," Mechanical Systems and Signal Processing, vol. 49, pp. 264-284, 2014.

[17] M. L. Jonathan and C. O. Sofia, "Higher- Order Properties of Analytic Wavelets," IEEE Transactions on Signal Processing, vol. 57, no. 1, pp. 146-160, 2008.

[18] S. Petre and M. Randolph, Spectral Analysis of Signals, Prentice Hall, Inc., 2005.

[19] T. Christopher and P. C. Gilbert, "A Practical Guide to Wavelet Analysis," Bulletin of the American Meteorological Society, vol. 79, no. 1, pp. 61-78, 1997.

[20] F. Zhipeng, L. Ming and C. Fulei, "Recent advances in time-frequency analysis methods for machinery fault diagnosis: A review with application examples," Mechanical Systems and Signal Processing, vol. 38, pp. 165-205, 2013.

[21] F. P.-R. Antonio and R. Rafael, "The convolution theorem for the continuous wavelet transform," Signal Processing, vol. 84, no. 1, pp. 55-67, 2004.

[22] S. Mallat and P. Gabriel, A Wavelet Tour of Signal Processing: The Sparse Way, Third Edition ed., Burlington: Elsevier, 2009.

[23] A. Kacha, F. Grenez and K. Benmahammed, "Time-Frequency Analysis of Multichannel Signals Using Two-Sided Autoregressive Modeling," Circuits, Systems \& Signal PRocessing, vol. 28, p. 309, 2008.

[24] C. J.A., "Spectral estimation: An overdetermined rational model equation approach," Proceedings of the IEEE, vol. 70, no. 9, pp. 907-939, 1982. 
[25] B. Boashash, "Estimating and Interpreting the instantaneous Frequency of a Signal --- Part 2: Algorithm and Applications," Proceedings of the IEEE, vol. 80, pp. 540-568, 1992.

[26] M. L. Jonathan, "Element analysis: a wavelet-based method for analysing time-localized events in noisy time series," Proc. R. Soc. A, vol. 473, no. 20160776, 2017.

[27] J. Antoni, J. Griffaton, H. Andre, L. Avendano-Valencia, F. Bonnardot, O. C.-- Morales, G. C.-. Dominguez, A. Daga, Q. Leclere, C. Vicuna, D. Acuna, A. Ompusunggu and E. Sierra-Alonso, "Feedback on the Surveillance 8 challenge: Vibration-based diagnosis of a Safran aircraft engine," Mechanical systems and Signal Processing, vol. 97, pp. 112-144, 2017. 
Figures
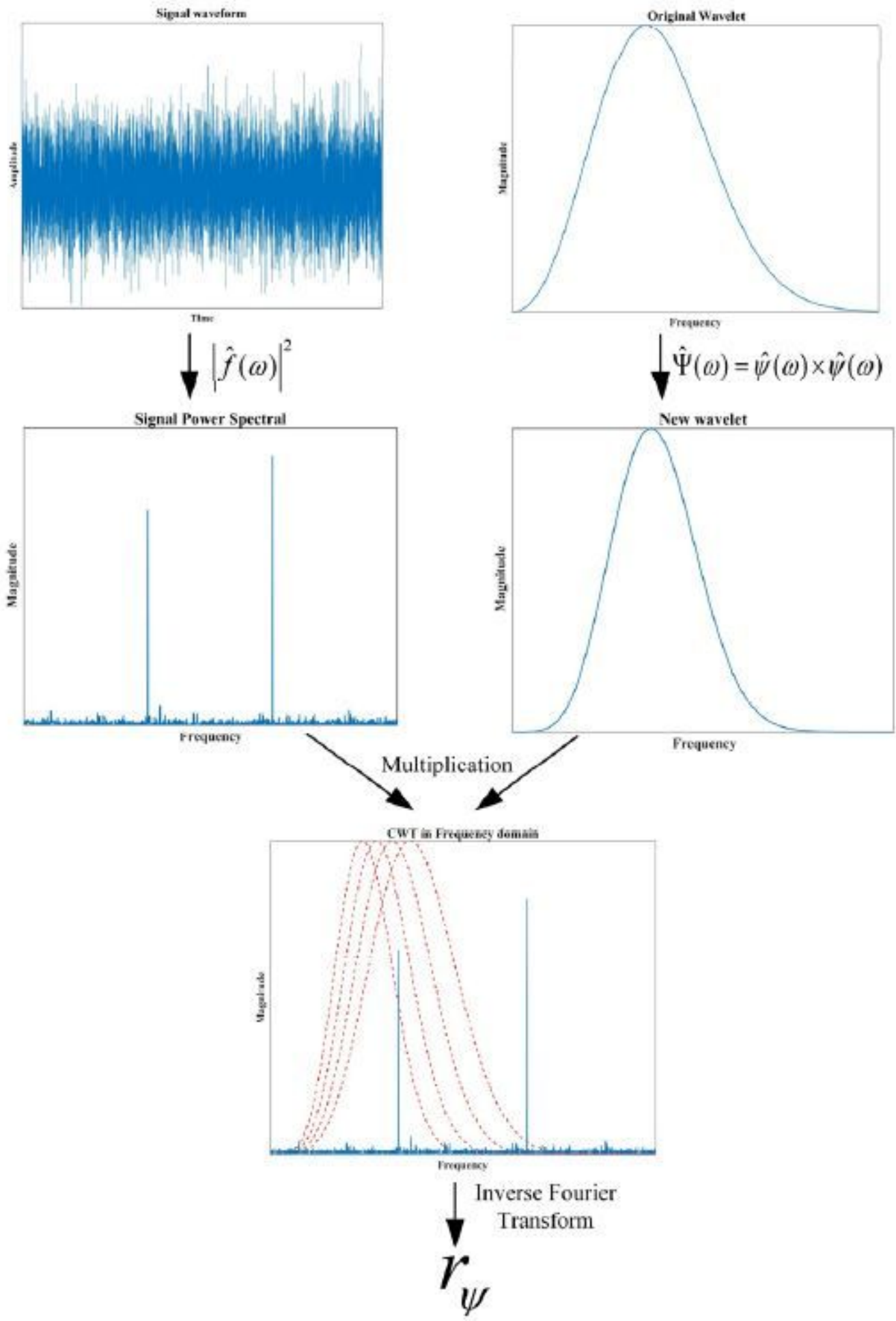

Figure 1

Illustration of the fast implementation 


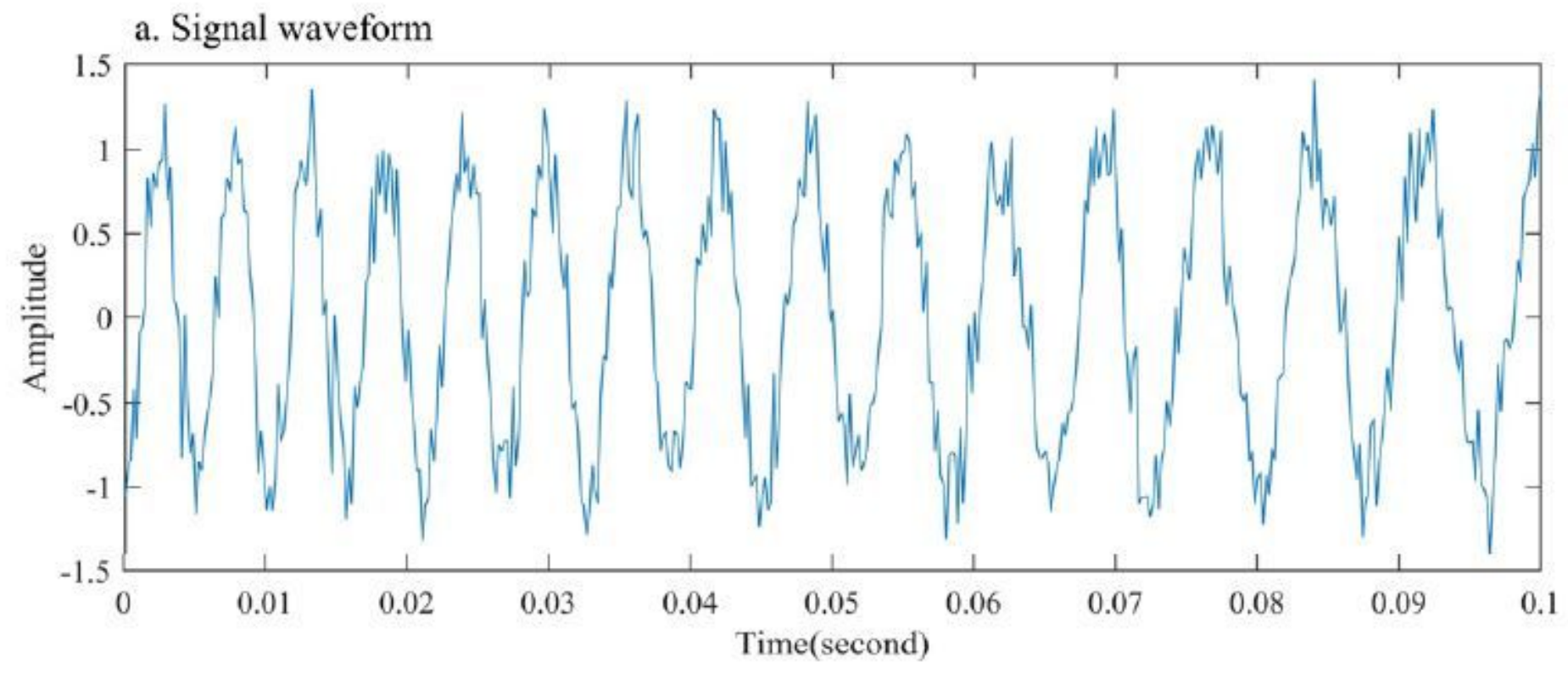

b. Signal spectrum

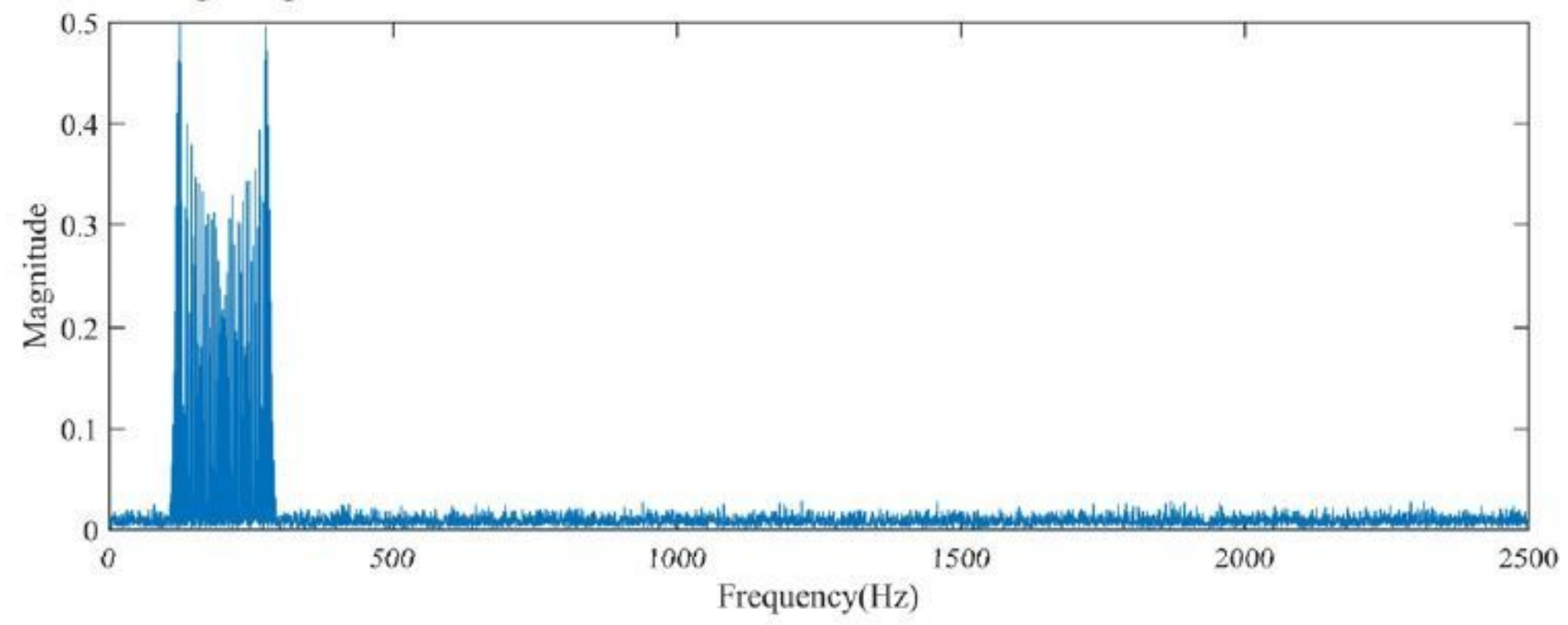

Figure 2

The synthetic signal. a. signal waveform. b. signal spectrum. 


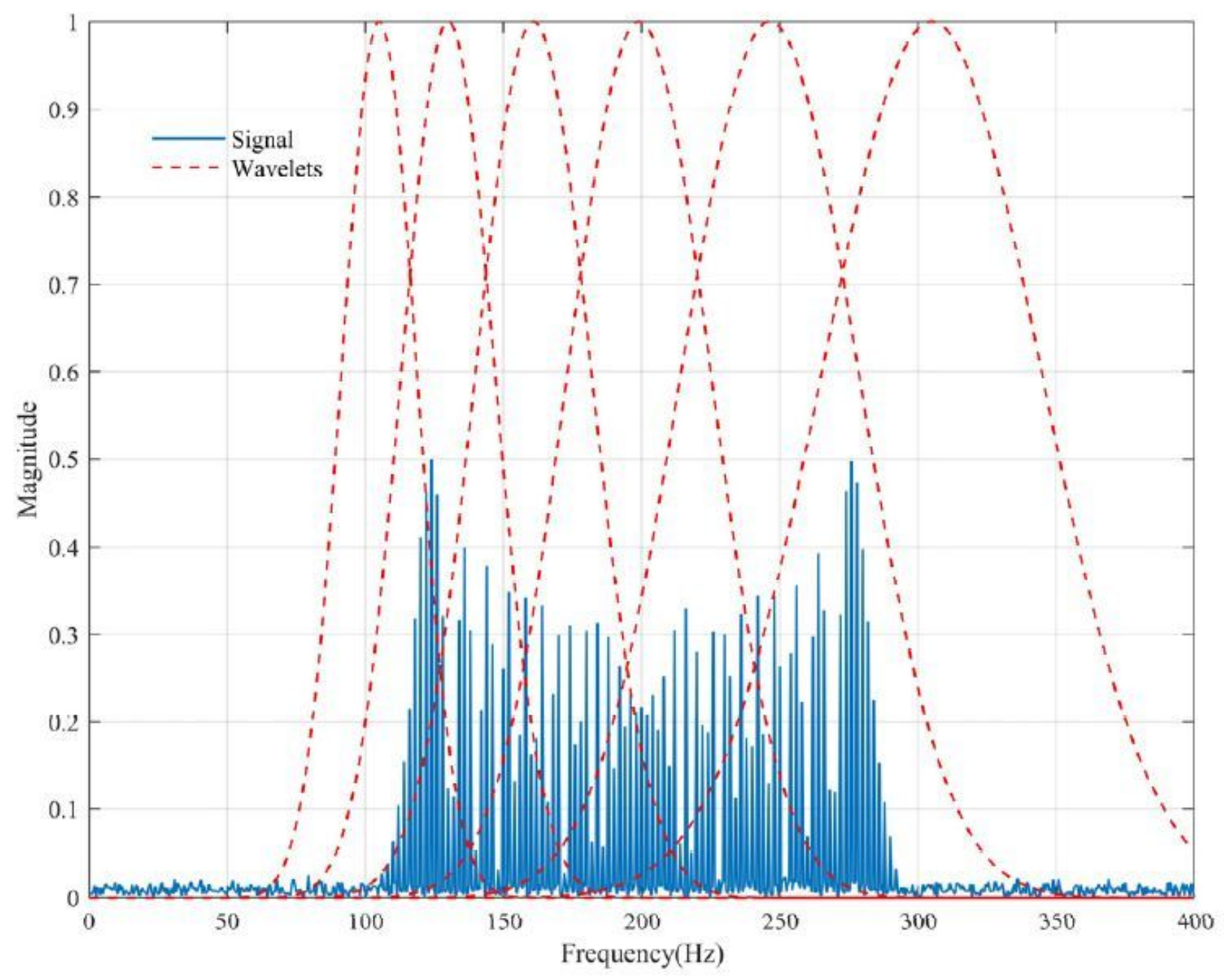

Figure 3

The filterbank. 


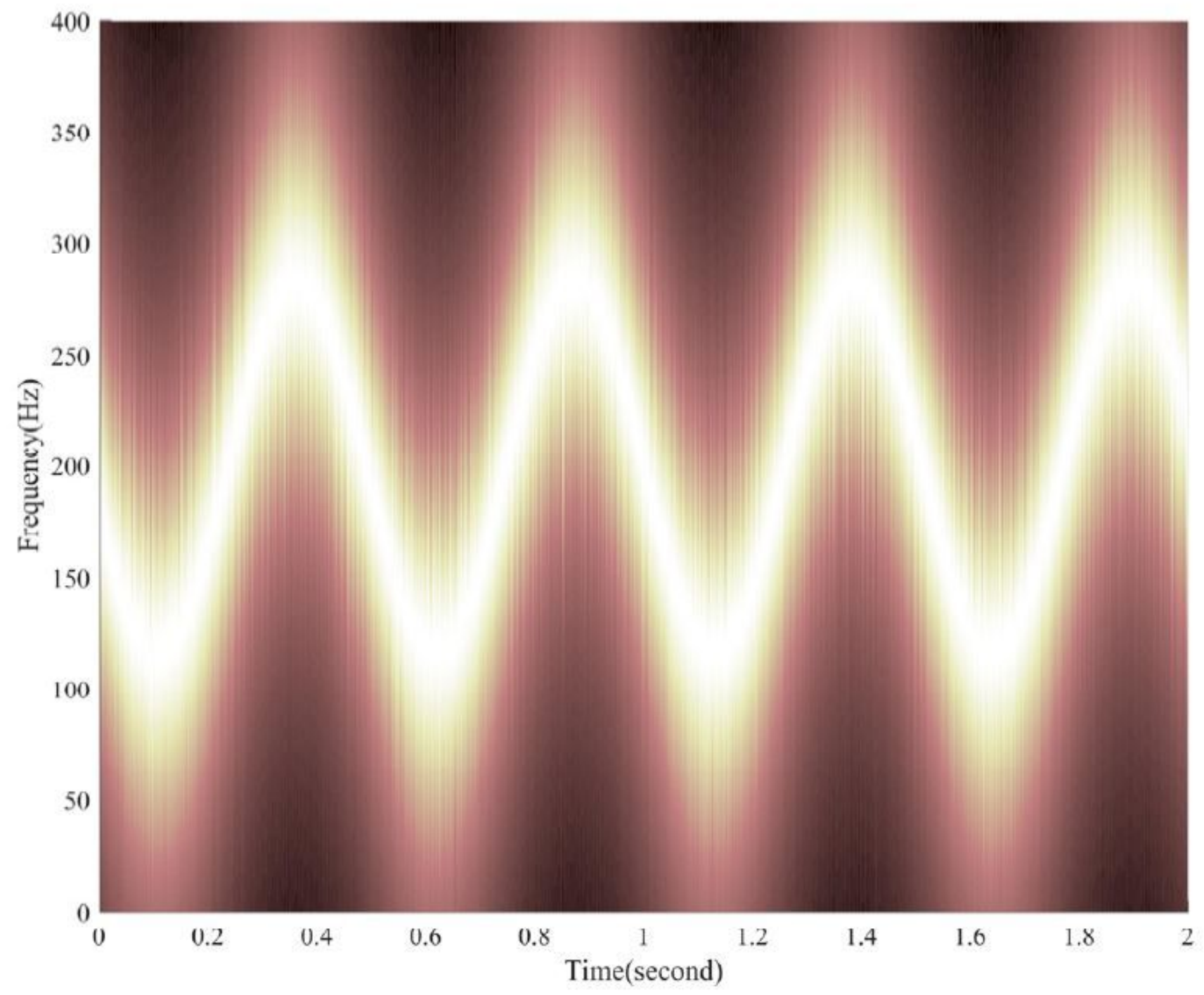

Figure 4

PSD of the signal. 


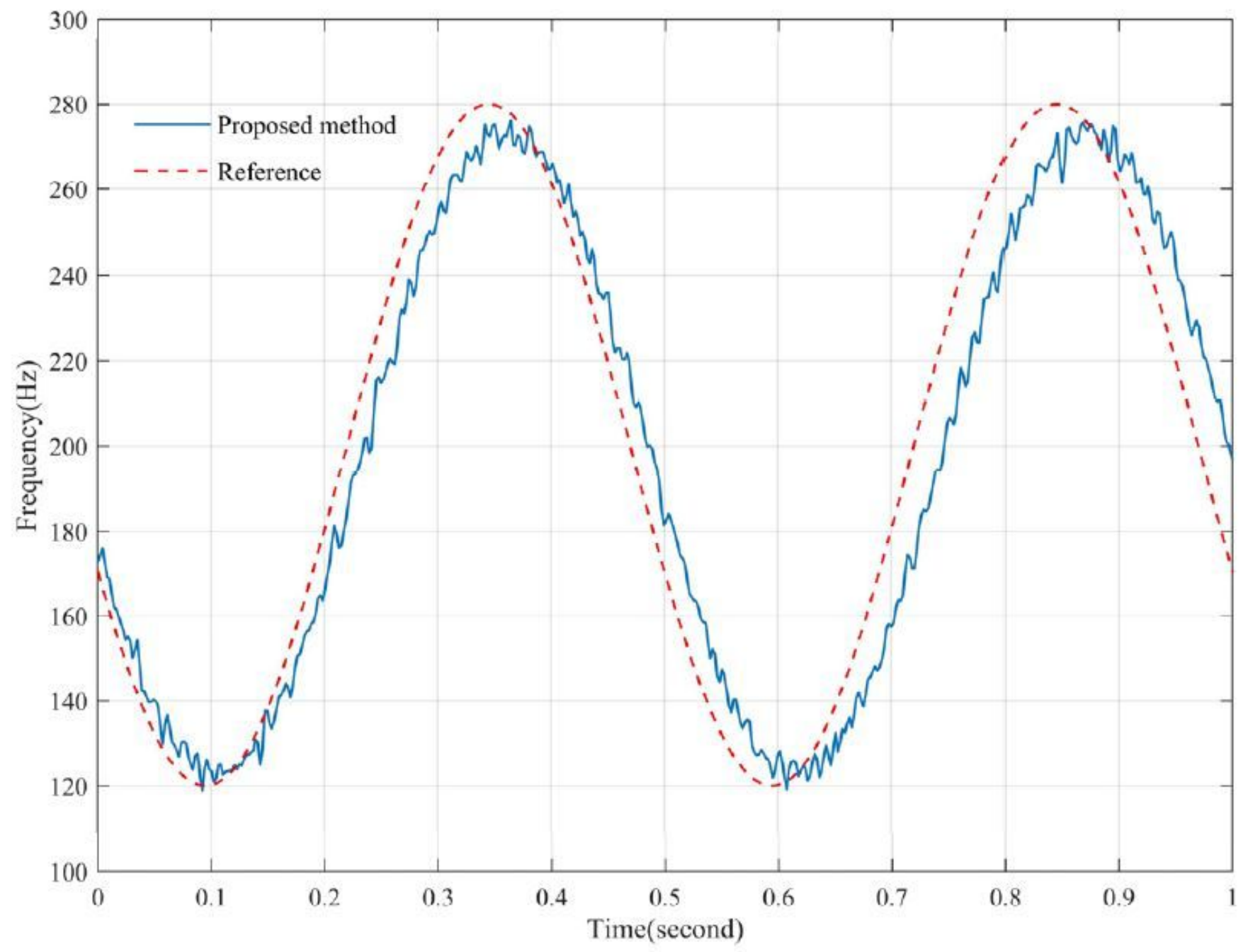

Figure 5

Estimated result of the proposed method. 


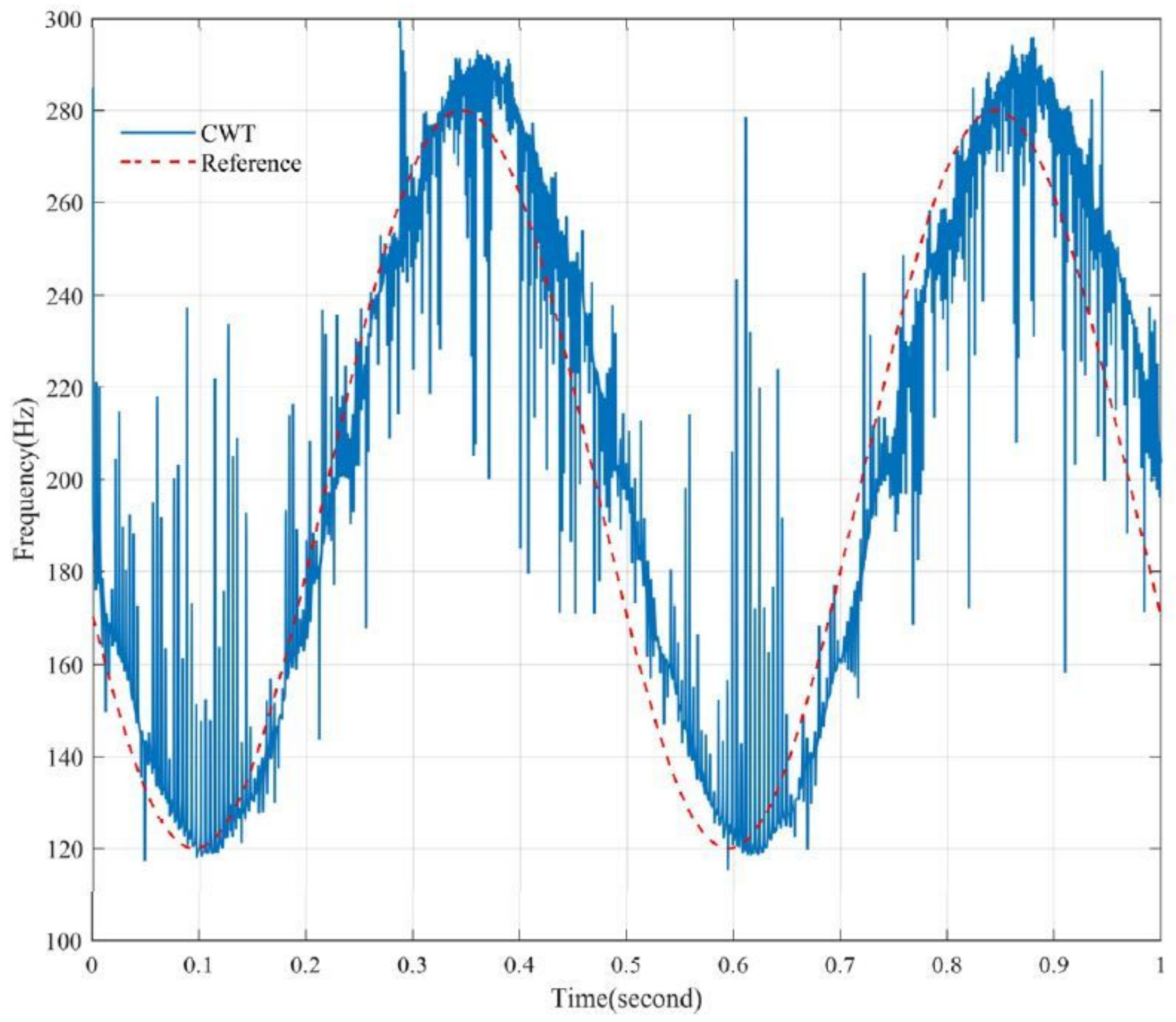

Figure 6

Estimated result of the CWT. 


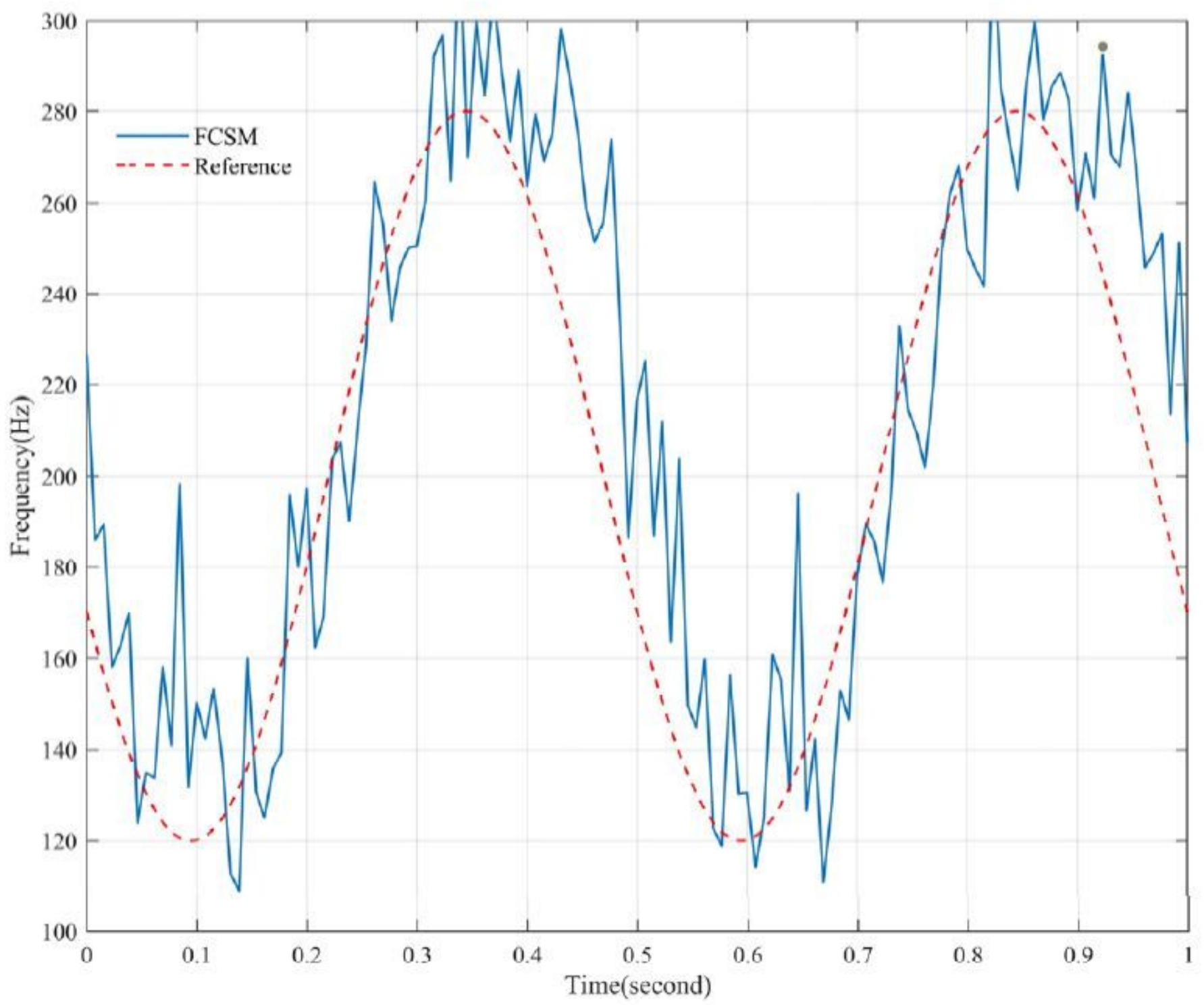

Figure 7

Estimated result of the FCSM. 


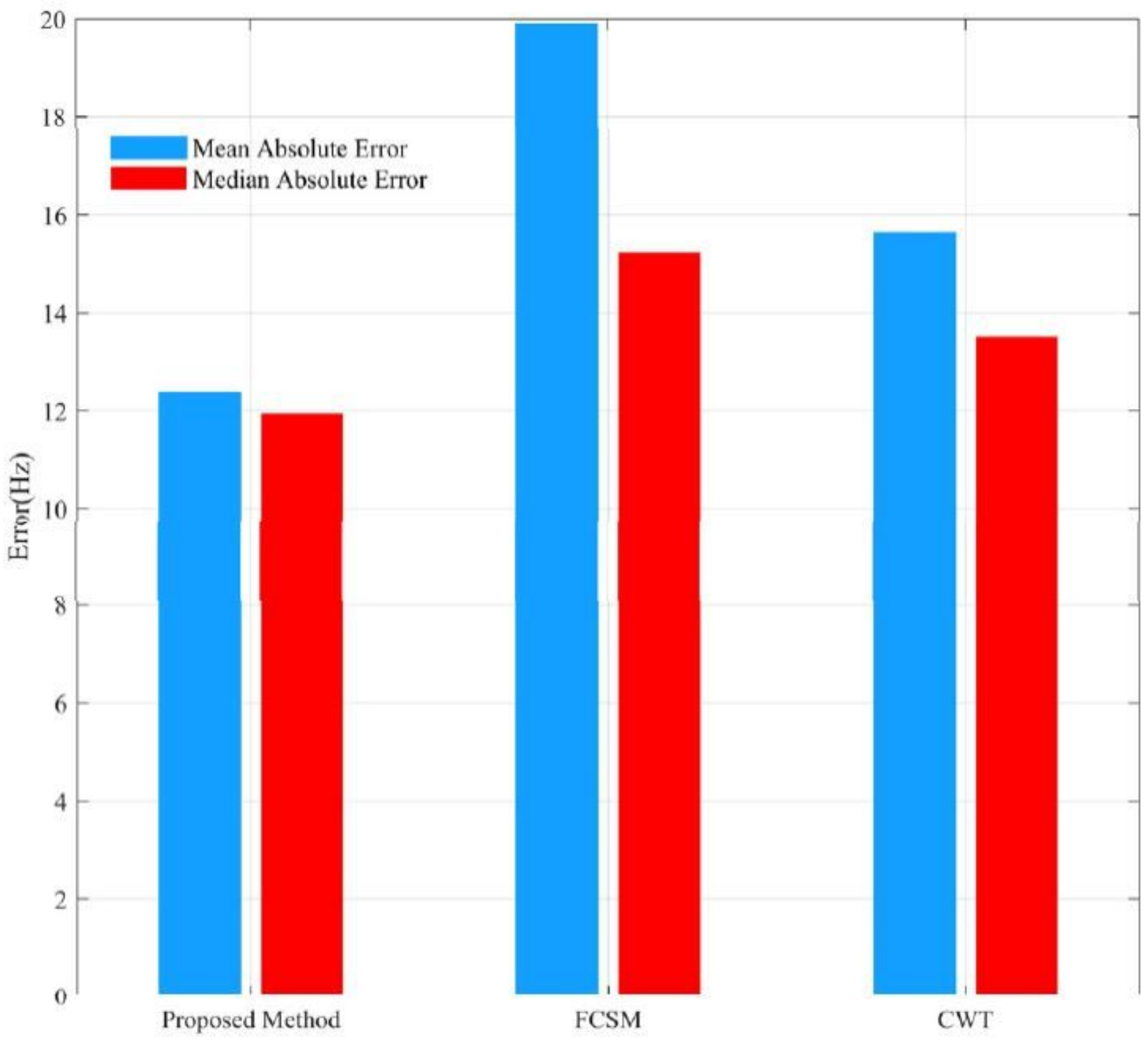

Figure 8

Mean and median absolute errors. 

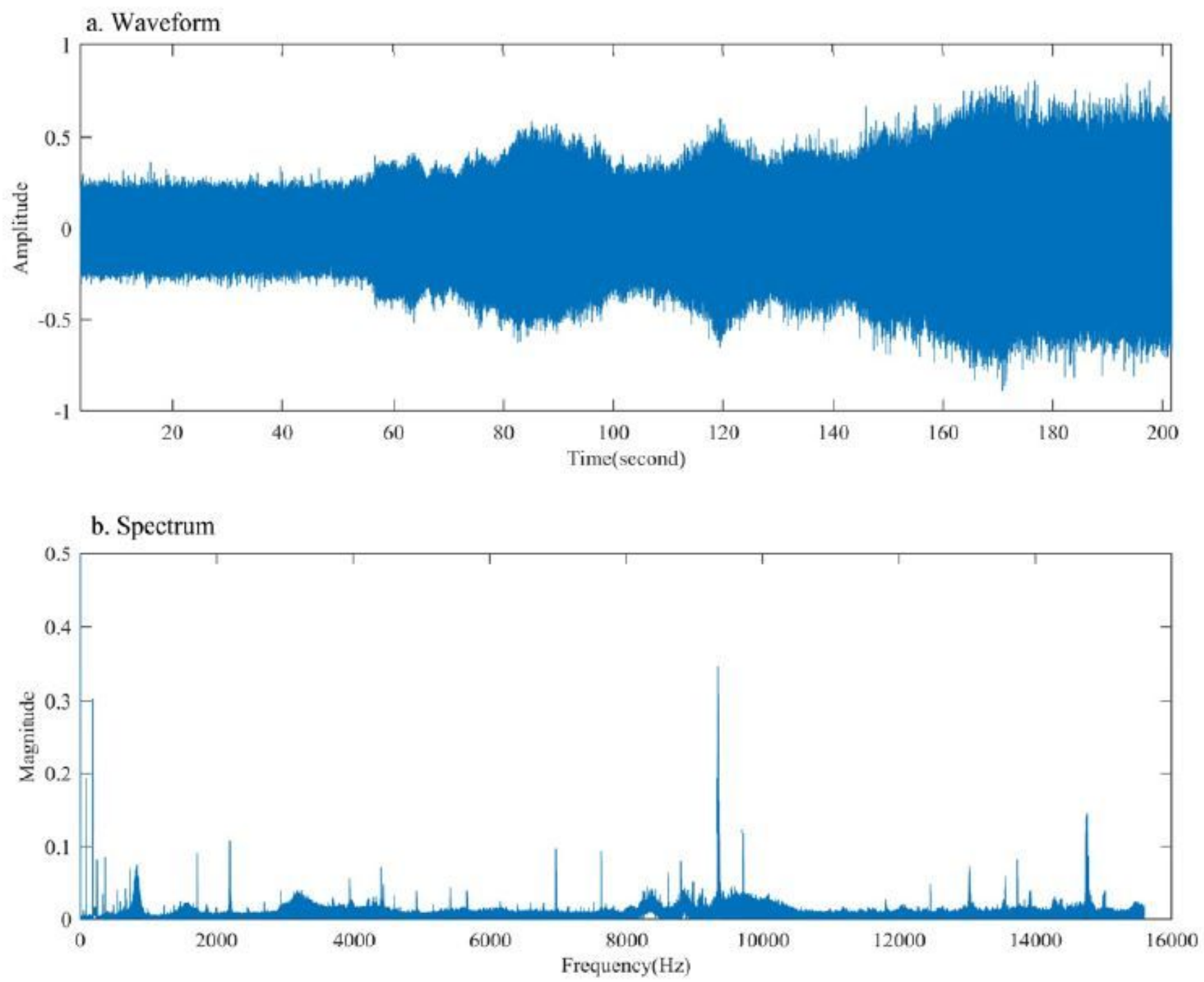

\section{Figure 9}

The vibration signal. a. signal waveform. b. signal spectrum. 


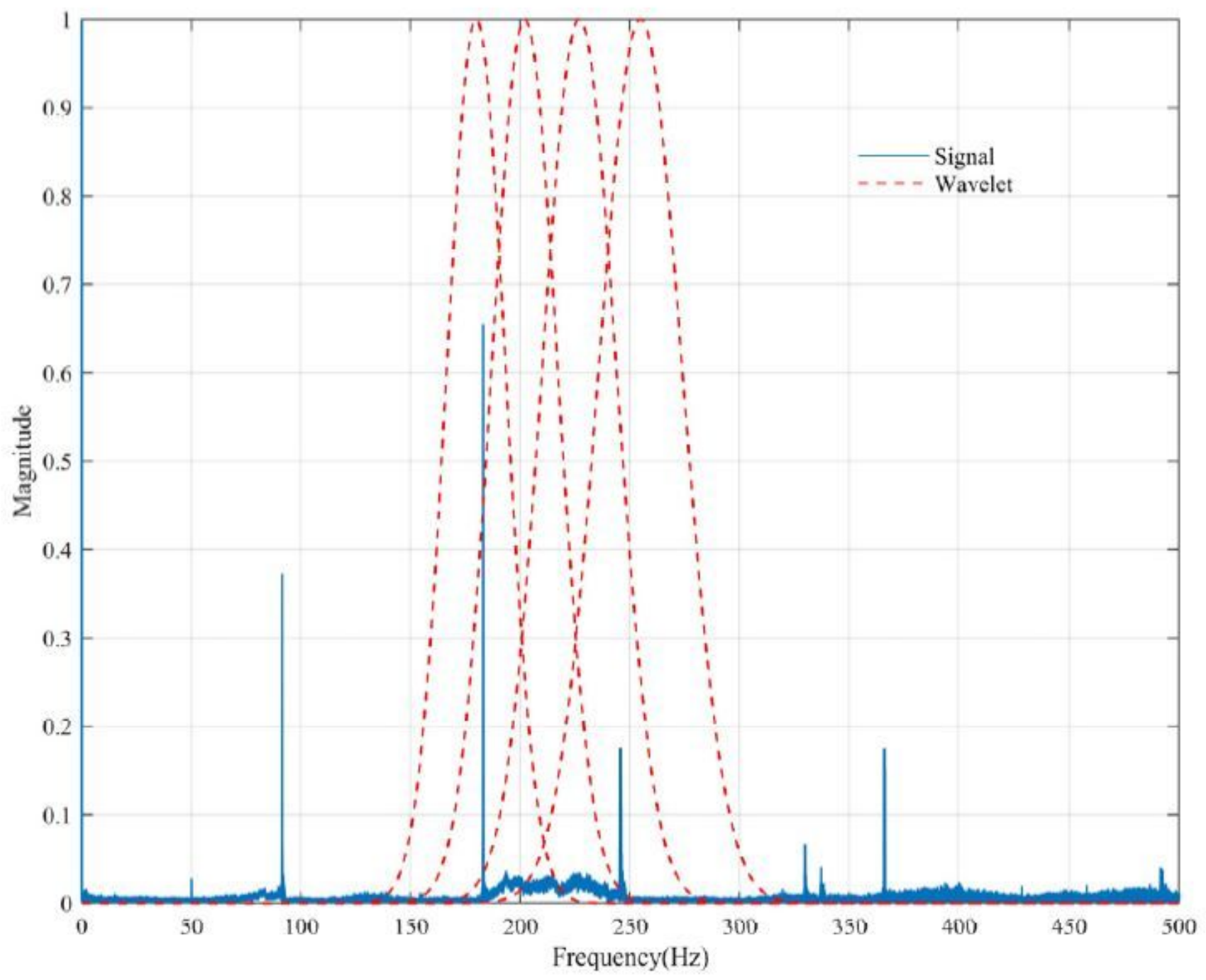

Figure 10

wavelet filterbank. 


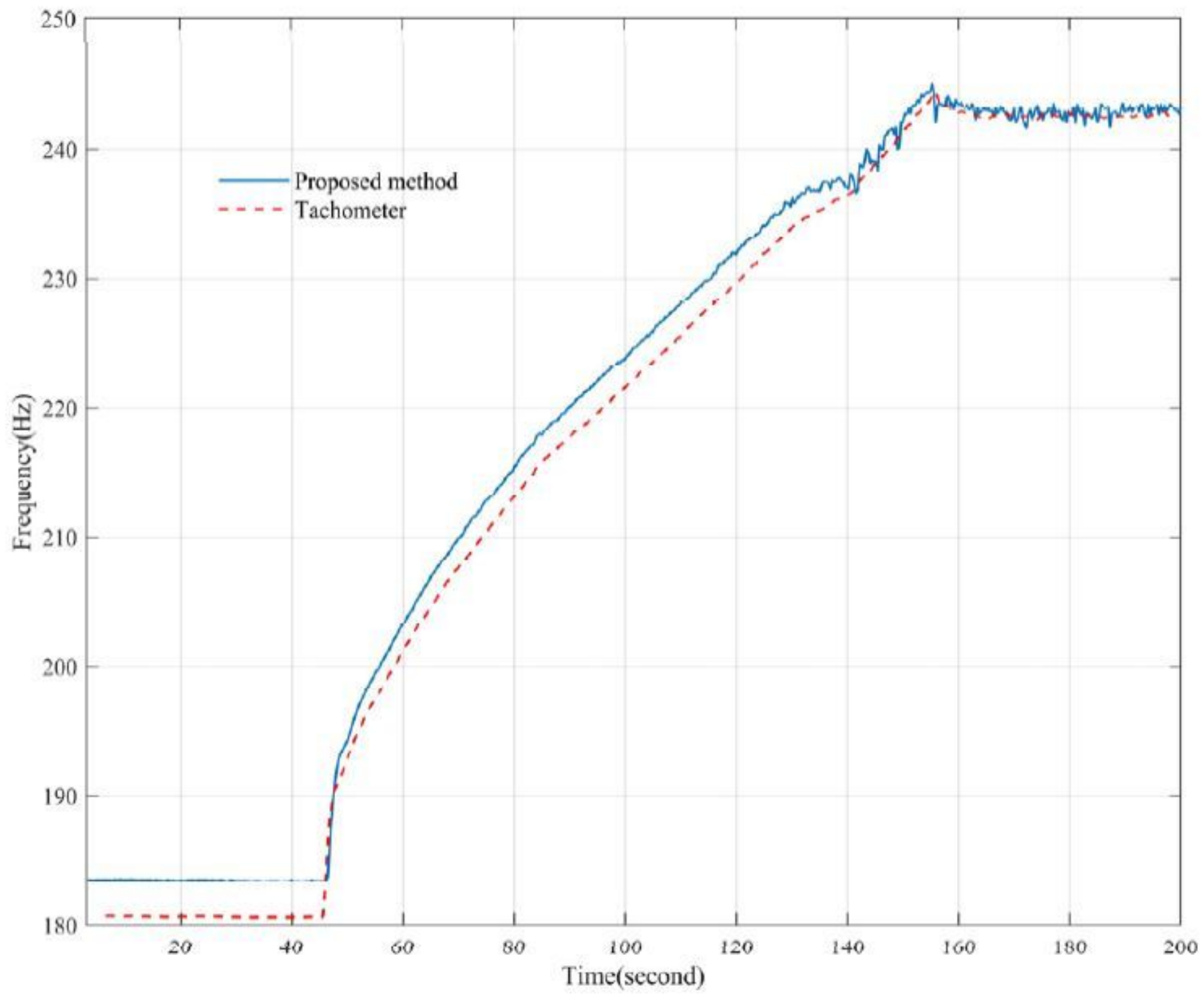

Figure 11

Estimated speed frequency of proposed method. 


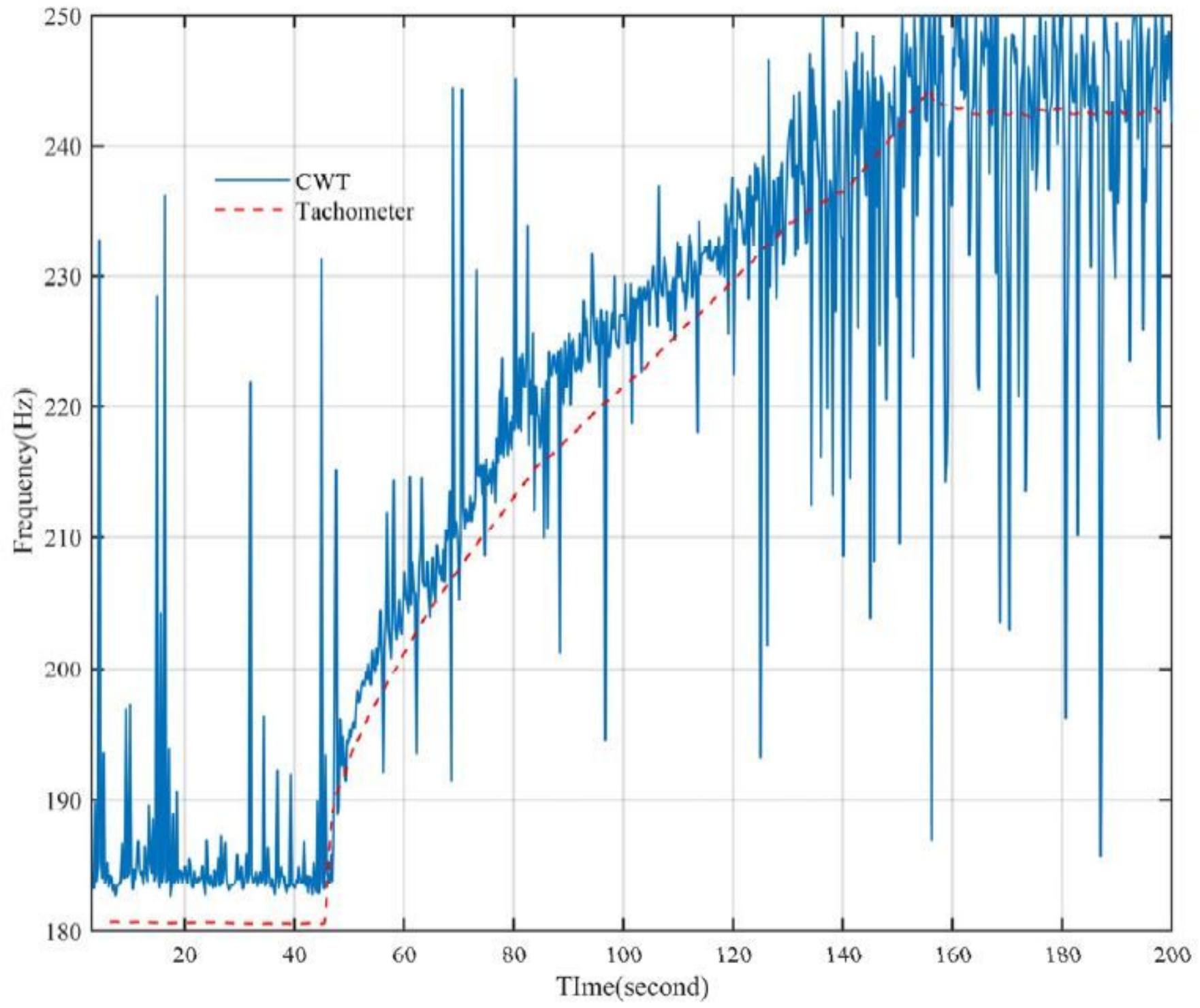

Figure 12

Estimated speed frequency of CWT. 


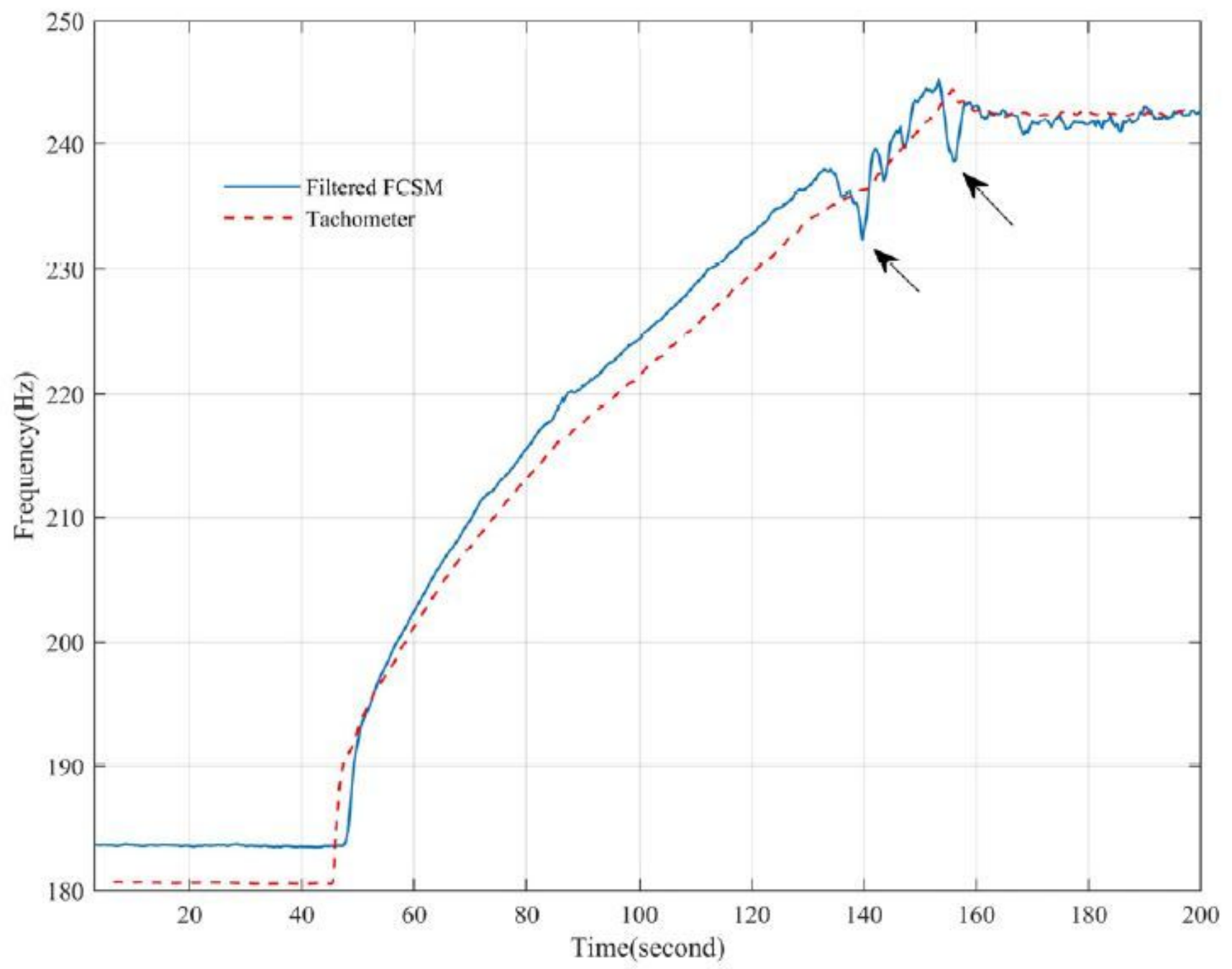

Figure 13

Estimated speed frequency of Filtered FCSM. 


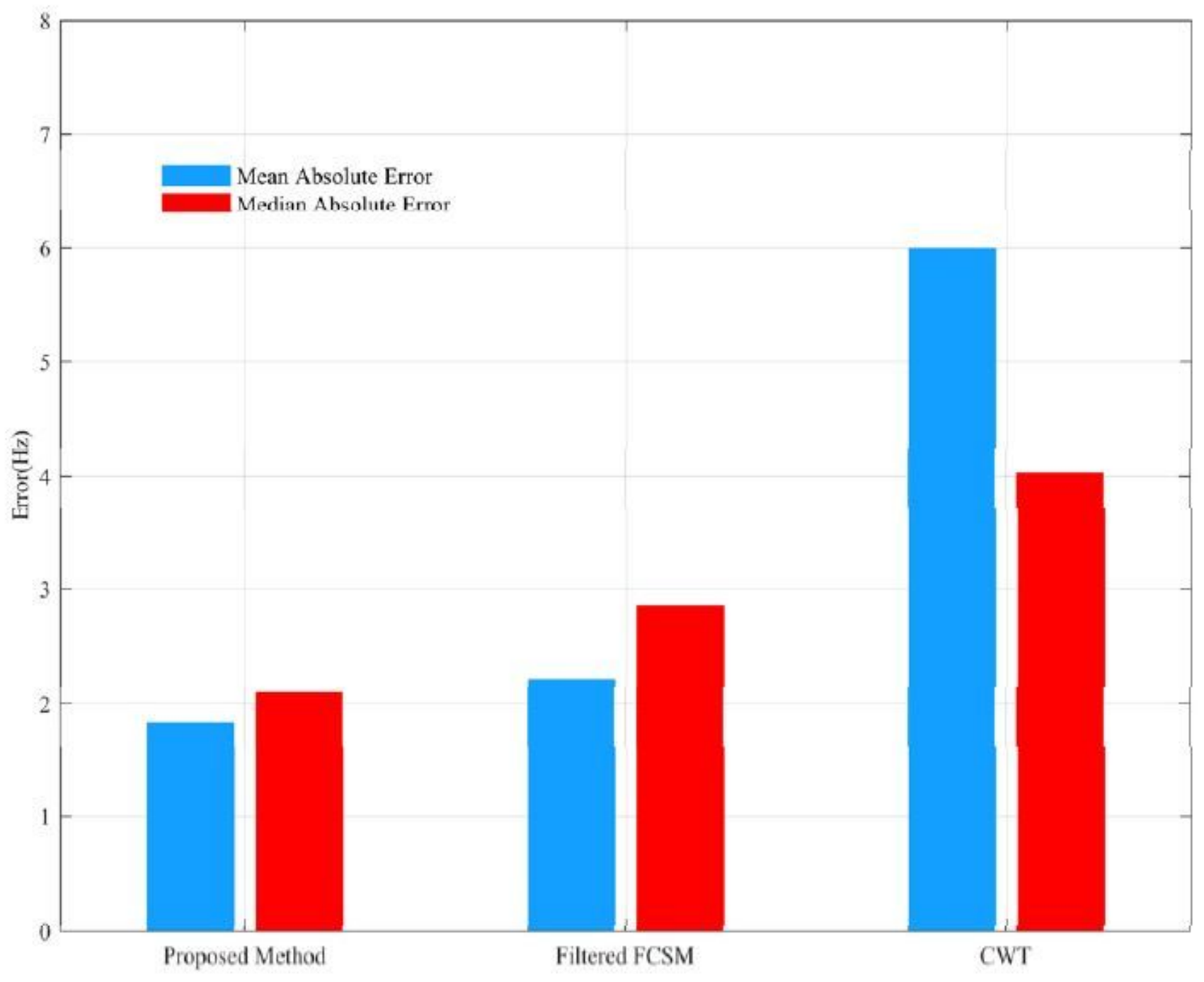

Figure 14

Mean and median absolute errors. 